胞 SpringerWien NewYork 


\title{
Kurzkommentar zum ABGB
}

\section{Allgemeines bürgerliches Gesetzbuch, Ehegesetz, Konsumentenschutzgesetz, IPR-Gesetz, Rom I- und Rom II-VO}

\author{
herausgegeben von \\ Helmut Koziol \\ Peter Bydlinski \\ Raimund Bollenberger \\ mit Beiträgen von \\ Peter Apathy Ernst Karner \\ Raimund Bollenberger Georg Kathrein \\ Michael Bydlinski Bernhard A. Koch \\ Peter Bydlinski Helmut Koziol \\ Karl-Heinz Danzl Franz Stefan Meissel \\ Wilma Dehn Gottfried Musger \\ Bernhard Eccher Matthias Neumayr \\ Irmgard Griss Andreas Riedler \\ Gerhard Hopf Hansjörg Sailer \\ Gert Iro Anton Spenling \\ Redaktionsassistenz: Susanne Haas \\ 3., überarbeitete und erweiterte Auflage \\ 2010

\section{SpringerWienNewYork}


Das Werk ist urheberrechtlich geschützt.

Die dadurch begründeten Rechte, insbesondere die der Übersetzung, des Nachdruckes, der Entnahme von Abbildungen, der Funksendung, der Wiedergabe auf photomechanischem oder ähnlichem Wege und der Speicherung in Datenverarbeitungsanlagen, bleiben, auch bei nur auszugsweiser Verwertung, vorbehalten. Die Wiedergabe von Gebrauchsnamen, Handelsnamen, Warenbezeichnungen usw. in diesem Buch berechtigt auch ohne besondere Kennzeichnung nicht zu der Annahme, dass solche Namen im Sinne der Warenzeichen- und Markenschutz-Gesetzgebung als frei zu betrachten wären und daher von jedermann benutzt werden dürfen.

Produkthaftung: Sämtliche Angaben in diesem Fachbuch/wissenschaftlichen Werk erfolgen trotz sorgfältiger Bearbeitung und Kontrolle ohne Gewähr. Eine Haftung der Autoren, der Herausgeber oder des Verlages aus dem Inhalt dieses Werkes ist ausgeschlossen.

(C) 2005, 2007 und 2010 Springer-Verlag/Wien

Printed in Germany

SpringerWienNew York ist ein Unternehmen von

Springer Science + Business Media

springer.at

Satz: Jung Crossmedia Publishing GmbH, 35633 Lahnau, Deutschland Druck: Druckerei C. H. Beck, 86720 Nördlingen, Deutschland

Gedruckt auf säurefreiem, chlorfrei gebleichtem Papier SPIN: 12722754

Bibliografische Information der Deutschen Nationalbibliothek Die Deutsche Nationalbibliothek verzeichnet diese Publikation in der Deutschen Nationalbibliografie; detaillierte bibliografische Daten sind im Internet über http://dnb.d-nb.de abrufbar. 


\section{Autorenverzeichnis}

Dr. Peter Apathy, o. Univ.-Prof. Universität Linz (peter.apathy@jku.at): S\531-796, 1045-1089 ABGB

Dr. Raimund Bollenberger, Rechtsanwalt bei Doralt - Seist - Csoklich, Univ.-Prof. WU Wien (raimund.bollenberger@wu.ac.at): \$S 859-880, 901-916, 938-956 ABGB

Dr. Michael Bydlinski, Hofrat des Obersten Gerichtshofs, Univ.-Prof. Universität Wien (michael.bydlinski@justiz.gv.at): $\mathbb{\$} \$ 1165-1173$ ABGB

Dr. Peter Bydlinski, o. Univ.-Prof. Universität Graz (peter.bydlinski@ uni-graz.at): $\$ \$ 1-14,880 a-900,917-937,1002-1034,1342-1367$ ABGB

Dr. Karl-Heinz Danzl, Senatspräsident des Obersten Gerichtshofs, Hon.-Prof. Universität Innsbruck (karl-heinz.danzl@justiz.gv.at): S\$1316-1341 ABGB

Dr. Wilma Debn, Richterin des OLG Wien (wilma.dehn@justiz.gv.at): $\$ \$ 1478-1502$ ABGB

Dr. Bernhard Eccher, o. Univ.-Prof. Universität Innsbruck (bernhard. eccher@uibk.ac.at): $\$ \int 285-446$ ABGB

Dr. Irmgard Griss, LL.M. (Harvard), Präsidentin des Obersten Gerichtshofs, Hon.-Prof. Universität Graz (ig@griss.at): \$\$ 957-1000, 1438-1450 ABGB

Dr. Gerhard Hopf, Sektionschef im Bundesministerium für Justiz i.R., Hon.-Prof. Universität Graz (gerhard.hopf@gmx.at): $\mathbb{\$} \mathbb{1 3 7 - 2 8 4 h}$ ABGB

Dr. Gert Iro, Univ.-Prof. Universität Wien (gert.iro@univie.ac.at): S\$1090-1121 ABGB

Dr. Ernst Karner, ao. Univ.-Prof. Universität Wien, Institut für Europäisches Schadenersatzrecht der Österreichischen Akademie der Wissenschaften (ernst.karner@univie.ac.at): \$\$ 1267-1315 ABGB 


\section{Autorenverzeichnis}

Dr. Georg Kathrein, Sektionschef im Bundesministerium für Justiz, Hon.-Prof. Universität Wien (georg.kathrein@bmj.gv.at): $\$ \$ \$ 1-32$ KSchG

Dr. Bernhard A. Koch, Univ.-Prof., LL.M. (Michigan), Universität Innsbruck (bernhard.a.koch@uibk.ac.at): $\mathbb{\$} \$ 15-100,447-530,1217-$ 1266, $1368-1374$ ABGB, $\$ \$ \$ 1-104$ EheG

Dr. Dr.hc. Helmut Koziol, o. Univ.-Prof. i.R. Universität Wien, Hon.Prof. Universität Graz, Direktor-Stellvertreter des Instituts für Europäisches Schadenersatzrecht der Österreichischen Akademie der Wissenschaften und Direktor des European Centre of Tort and Insurance Law (koziol@ectil.org): \$\$ 1035-1044, 1174, 1411-1437 $\mathrm{ABGB}$

Dr. Franz Stefan Meissel, Univ.-Prof. Universität Wien (franz.stefan. meissel@univie.ac.at): \$\$ 1451-1477 ABGB

Dr. Gottfried Musger, Hofrat des Obersten Gerichtshofs (gottfried. musger@justiz.gv.at): Art 1-29 Rom I-VO

Dr. Matthias Neumayr, Hofrat des Obersten Gerichtshofs, Hon.-Prof. Universität Linz (matthias.neumayr@justiz.gv.at): $\mathbb{\$} \mathbb{\$} 1375-1410$ ABGB, $\mathbb{S} \mathbb{S} 1-54$ IPRG, Art 1 - 32 Rom II-VO

Dr. Andreas Riedler, Univ.-Prof. Universität Linz (andreas.riedler@jku. at): $\$ \$ \$ 1175-1216$ ABGB

Dr. Hansjörg Sailer, Hofrat des Obersten Gerichtshofs, Hon.-Prof. Universität Linz (hansjoerg.sailer@aon.at): \$S 797-858 ABGB

Dr. Anton Spenling, Senatspräsident des Obersten Gerichtshofs (anton. spenling@justiz.gv.at): \$\$ 1151-1164a ABGB 


\section{Vorwort zur 3. Auflage}

Diese dritte Auflage erscheint etwa drei Jahre nach der zweiten. Sie wurde nicht nur zwecks Einarbeitung neuester Judikatur und Literatur erforderlich, sondern auch weil der Gesetzgeber wieder vielfältig tätig war: das Darlehens- und Kreditrecht wurde neu gestaltet (BGBl I 2010/ 28), das Familienrecht (BGBl I 2009/75) und das Insolvenzrecht geändert (BGBl I 2010/29 und 2010/58) und die gleichgeschlechtlichen Partnerschaften (BGBl I 2009/135) geregelt.

Ferner haben sich die Herausgeber wiederum für eine maßvolle Erweiterung des behandelten Rechtsstoffs entschieden: Es sind prägnante Kommentierungen der international-privatrechtlichen EG-Verordnungen Rom I und Rom II, verfasst durch Richter des OGH, hinzugekommen. Damit ist für die nächste Zeit die Erweiterung des Buches abgeschlossen. Publikationen wurden grundsätzlich bis April 2010 berücksichtigt; einzelne besonders wichtige konnten bis Ende Juli nachgetragen werden.

Am bewährten Konzept des „KBB“, der sich nunmehr auf dem Gesetzesstand vom 1.8.2010 befindet, wurde nichts geändert.

Für das pünktliche Erscheinen der dritten Auflage haben wir wiederum ganz besonders unserer „Redaktionsassistentin“, Frau RiAA Mag. Susanne Haas, zu danken. Sie hat sich mit außergewöhnlichem Einsatz, großer Umsicht, beispielhafter Genauigkeit und erfreulicher Selbständigkeit um alle „Formalien“ gekümmert. Der Verlag war wie gewohnt bemüht, das rasche Erscheinen zu ermöglichen. Um die gründliche Überarbeitung des Sachregisters hat sich Herr WissMi Mag. Stephan Foglar-Deinharstein verdient gemacht. Ihnen allen sowie so manchem ungenannten Helfer im Hintergrund sei auch an dieser Stelle herzlicher Dank gesagt. Nicht zuletzt aber danken wir aufrichtig unseren engagierten Autorinnen und Autoren, die sich nicht nur erfolgreich um die in- 


\section{Vorwort zur 3. Auflage}

haltsreiche Kommentierung bemühen, sondern geduldig und verständnisvoll auch den peinigenden Wünschen der Herausgeber betreffend die Einheitlichkeit und Kürze des Werkes nachkommen. Es hat wiederum Freude gemacht, mit diesem Team zu arbeiten!

Wien/Graz, im Juli 2010

Die Herausgeber 


\section{Aus dem Vorwort der 1. Auflage}

Das ABGB und einige wichtige Nebengesetze sind schon seit längerem durch mehrere Kommentare größeren Umfangs erschlossen; ebenso durch Sammlungen von Leitsätzen höchstgerichtlicher Entscheidungen. Ein Kurzkommentar, der vor allem dem Praktiker, aber auch dem fortgeschrittenen Studenten, systematische und aktuelle Erstinformation verschafft, fehlte in Österreich jedoch bisher. Herausgeber und Verlag sind der Meinung, dass es am Beginn des 21. Jahrhunderts hoch an der Zeit ist, diese Lücke zu schließen.

Natürlich muss ein solches Werk - und damit auch sein Benutzer - mit manchen Unvollständigkeiten leben. So konnten neben dem ABGB nur Ehegesetz und Konsumentenschutzgesetz aufgenommen werden; andere wichtige Rechtsquellen kommen in der Kommentierung aber selbstverständlich ebenfalls zur Sprache. Das Konzept des Kurzkommentars ist rasch erklärt: Der Benutzer soll sowohl über die zentralen Grundsätze von Rechtsinstituten und Einzelbestimmungen als auch über die wichtigsten Detailfragen informiert werden. Im Vordergrund steht dabei die höchstgerichtliche Judikatur. Die Anführung der Geschäftszahl bei den seit 1990 ergangenen Entscheidungen dient der erleichterten elektronischen Recherche. Trotz der konzeptionell vorgegebenen Knappheit der Kommentierung sollte sich die überwiegende Zahl der in der Alltagspraxis auftretenden Rechtsfragen bereits mit dem vorliegenden Werk und der angeführten Judikatur lösen lassen. Ansonsten helfen andere, im Kommentar zitierte Publikationen. Bei der oft schwierigen - Literaturauswahl wurde den Aspekten Aktualität, Greifbarkeit und weiterführender Problemaufbereitung besonderer Stellenwert eingeräumt. Die Selbstbeschränkung eines Kurzkommentars hat für den Benutzer weitere handfeste Vorteile: Das Buch passt in jede Aktentasche, es ist für jedermann erschwinglich und Neuauflagen 


\section{Aus dem Vorwort der 1. Auflage}

können in relativ kurzen Abständen erfolgen, so dass das Werk aktuell bleibt.

Das vor etwa vier Jahren zusammengestellte und seither nur geringfügig veränderte Autorenteam besteht neben Universitätslehrern aus Richtern (vor allem des Obersten Gerichtshofs), einem Rechtsanwalt und aus Spitzenbeamten des Justizministeriums. 


\section{Inhaltsverzeichnis}

Benutzerhinweise ................. XVII Abgekürzt zitierte Literatur $\ldots \ldots \ldots \ldots \ldots \ldots \ldots$ XIX Abkürzungsverzeichnis $\ldots \ldots \ldots \ldots \ldots \ldots \ldots \ldots \ldots$ XXXI

\section{Allgemeines bürgerliches Gesetzbuch}

Einleitung. Von den bürgerlichen Gesetzen überhaupt. $\mathbb{S} \mathbb{S 1 - 1 4}$

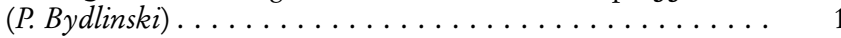

\section{Erster Teil. Von dem Personenrechte}

Erstes Hauptstück. Von den Rechten, welche sich auf persönliche Eigenschaften und Verhältnisse beziehen.

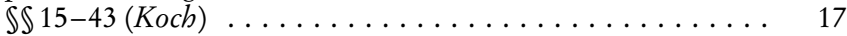

Zweites Hauptstück. Von dem Eherechte. $\$ \$$ 44-136 (Koch) . . . 39

Drittes Hauptstück. Von den Rechten zwischen Eltern und Kindern. $\$ \$ \$ 137-186 a$ (Hopf) . . . . . . . . . . . . . . 69

Viertes Hauptstück. Von der Obsorge einer anderen Person. $\$ \mathbb{S} 187-267$ (Hopf) . . . . . . . . . . . . . . . . . . . 196

Fünftes Hauptstück. Von der Sachwalterschaft, der sonstigen gesetzlichen Vertretung und der Vorsorgevollmacht.

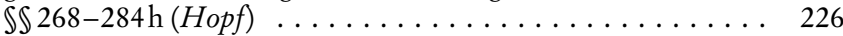

\section{Zweiter Teil. Von dem Sachenrechte}

Von Sachen und ihrer rechtlichen Einteilung. $\$ \mathbb{S} 285-308$

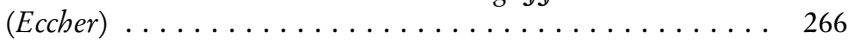




\section{Inhaltsverzeichnis}

\section{Erste Abteilung des Sachenrechtes. Von den dinglichen Rechten}

Erstes Hauptstück. Von dem Besitze. $\$ \$ 309-352$ (Eccher) . . . .

Zweites Hauptstück. Von dem Eigentumsrechte. $\$ \$ 353-379$

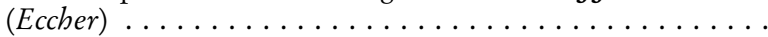

Drittes Hauptstück. Von der Erwerbung des Eigentumes durch Zueignung. $\int \mathbb{S} 380-403$ (Eccher) . . . . . . . . . . . . .

Viertes Hauptstück. Von Erwerbung des Eigentumes durch Zuwachs. $\$ \$ \$ 404-422$ (Eccher) ..................

Fünftes Hauptstück. Von Erwerbung des Eigentumes durch Übergabe. $\$ \$ \$ 423-446$ (Eccher) . . . . . . . . . . . . . .

Sechstes Hauptstück. Von dem Pfandrechte. $\int \mathbb{S} 447-471$ (Koch) . Siebentes Hauptstück. Von Dienstbarkeiten (Servituten).

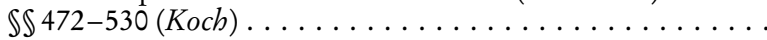

Achtes Hauptstück. Von dem Erbrechte. $\int \$ 531-551$ (Apathy) . .

Neuntes Hauptstück. Von der Erklärung des letzten Willens überhaupt und den Testamenten insbesondere. $\$ \$ \$ 552-603$

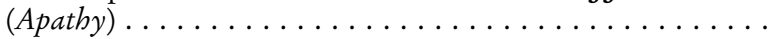

Zehntes Hauptstück. Von Nacherben [und Fideikommissen]. $\$ \$ \$ 604-646($ Apathy) . . . . . . . . . . . . . . . . . . . 560

Elftes Hauptstück. Von Vermächtnissen. \$\$ 647-694 (Apathy) . . 576

Zwölftes Hauptstück. Von Einschränkung und Aufhebung des letzten Willens. $\$ \$ \$ 695-726($ Apathy) . . . . . . . . . . . . .

Dreizehntes Hauptstück. Von der gesetzlichen Erbfolge. $\$ \$ \$ 727-761$ (Apathy) . . . . . . . . . . . . . . .

Vierzehntes Hauptstück. Von dem Pflichtteile und der Anrechnung in den Pflicht- oder Erbteil. \$\$ 762-796 (Apathy)

Fünfzehntes Hauptstück. Von Besitznehmung der Erbschaft. \$S 797-824 (Sailer) . . . . . . . . . . . . . . . . . . Sechzehntes Hauptstück. Von der Gemeinschaft des Eigentumes und anderer dinglichen Rechte. $\$ \$ \$ 825-858$ (Sailer) $\ldots \ldots$. .

\section{Zweite Abteilung. Von den persönlichen Sachenrechten}

Siebzehntes Hauptstück. Von Verträgen und Rechtsgeschäften überhaupt. \$\$ 859-937 (Bollenberger/P. Bydlinski) . . . . . . . .

Achtzehntes Hauptstück. Von Schenkungen. \$\$ 938-956

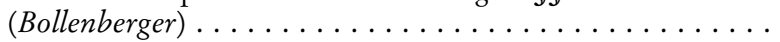

Neunzehntes Hauptstück. Von dem Verwahrungsvertrage. $\$ \mathbb{S} 957-970$ c (Griss) . . . . . . . . . . . . . . . . . . . . . 1041

Zwanzigstes Hauptstück. Von dem Leihvertrage. $\int \$ \$ 971-982$ (Griss) . . . . . . . . . . . . . . . . . . 1056

Einundzwanzigstes Hauptstück. Von dem Darlehensvertrage. $\$ \$ 983-1000$ (Griss) 
Zweiundzwanzigstes Hauptstück. Von der Bevollmächtigung und andern Arten der Geschäftsführung. $\$ \$$ 1002-1044 (P. Bydlinski/Koziol) . . . . . . . . . . . . . . . . 1086

Dreiundzwanzigstes Hauptstück. Von dem Tauschvertrage. $\$ \mathbb{S} 1045-1052$ (Apatby) . . . . . . . . . . . . . . . 1162

Vierundzwanzigstes Hauptstück. Von dem Kaufvertrage. $\$ \$ 1053-1089$ (Apathy) . . . . . . . . . . . . . .

Fünfundzwanzigstes Hauptstück. Von Bestand-, Erbpacht- und

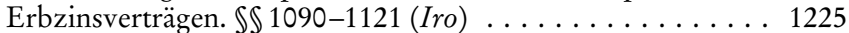

Sechsundzwanzigstes Hauptstück. Von Verträgen über Dienstleistungen. \$S1151-1174 (Spenling/M. Bydlinski/Koziol) ...

Siebenundzwanzigstes Hauptstück. Von dem Vertrage über eine Gemeinschaft der Güter. $\$ \$ \$ 1175-1216$ (Riedler) … . . . 1362 Achtundzwanzigstes Hauptstück. Von den Ehepakten und dem Anspruch auf Ausstattung. $\mathbb{S} \$ 1217-1266$ (Koch) . . . . . . 1408

Neunundzwanzigstes Hauptstück. Von den Glücksverträgen. $\mathbb{S} \$ 1267-1292$ (Karner) . . . . . . . . . . . . . . . . . . 1430

Dreißigstes Hauptstück. Von dem Rechte des Schadensersatzes und der Genugtuung. \$\$ 1293-1341 (Karner/Danzl) . . . . . 1446

\section{Dritter Teil. Von den gemeinschaftlichen Bestimmungen der Personen- und Sachenrechte}

Erstes Hauptstück. Von Befestigung der Rechte und Verbindlichkeiten. $\int \$ 1342-1374$ (P. Bydlinski/Koch) . . . . 1586

Zweites Hauptstück. Von Umänderung der Rechte und Verbindlichkeiten. $\$ \$ \$ 1375-1410$ (Neumayr) . . . . . . . 1631

Drittes Hauptstück. Von Aufhebung der Rechte und Verbindlichkeiten. $\$ \$ 1411-1450$ (Koziol/Griss) . . . . . . 1691 Viertes Hauptstück. Von der Verjährung und Ersitzung.

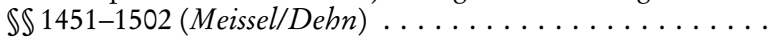

\section{Ehegesetz (Koch)}

\section{Erster Abschnitt. Recht der Eheschließung}

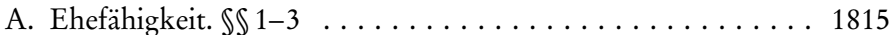

B. Eheverbote. $\int \mathbb{S} 6-10 \ldots \ldots \ldots \ldots \ldots \ldots \ldots \ldots 18 \ldots \ldots \ldots$

C. Eheschließung. $\$ \$ \$ 15-17 \ldots \ldots \ldots \ldots \ldots 18 \ldots$

D. Nichtigkeit der Ehe. $\mathbb{S} 20-32 \ldots \ldots \ldots \ldots \ldots \ldots 18 \ldots \ldots$

E. Aufhebung der Ehe. $\int \$ 33-42 \ldots \ldots \ldots \ldots \ldots \ldots$

F. Wiederverheiratung im Falle der Todeserklärung. $\$ \$$ 43-44 . 1837

G. Wiederverheiratung nach Auflösung der Vorehe durch eine ausländische Entscheidung. $\$ 45 \ldots \ldots \ldots \ldots \ldots 1838$ 


\section{Inhaltsverzeichnis}

\section{Zweiter Abschnitt. Recht der Ehescheidung}

A. Allgemeine Vorschriften. $\$ 46 \ldots \ldots \ldots \ldots \ldots 1838$

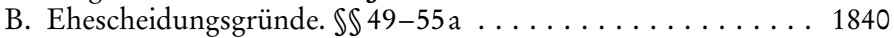

C. Ausschluß des Scheidungsrechts. $\$ \$ 56-59 \ldots \ldots \ldots 1853$

D. Schuldausspruch. $\mathbb{S} 560-61 \ldots \ldots \ldots \ldots \ldots \ldots$

E. Folgen der Scheidung. $\$ \mathbb{S} 62-98 \ldots \ldots \ldots \ldots \ldots$

\section{Dritter Abschnitt. Sondervorschriften für das Land} Österreich
A. Standesbeamte (aufgehoben)
B. Ergänzungsvorschriften. \$S 102-107
C. Verfahrensvorschriften (aufgeboben)
D. Übergangsbestimmungen. $\$ \$ 109-128$

Vierter Abschnitt. Schlußbestimmungen. $\$ \$ 129-130$

\section{Konsumentenschutzgesetz (Kathrein)}

\section{Hauptstück. Besondere Bestimmungen für Verträge zwischen Unternehmern und Verbrauchern}

Abschnitt I. Geltungsbereich. $\$ \int 1-2 \ldots \ldots \ldots$. . . . . . . . . . 1914 Abschnitt II. Allgemeine Regeln. $\$ \$ \$ 3-14 \ldots \ldots \ldots$. . . . . . . . 1920

Abschnitt III. Besondere Vertragsarten. $\$ \$ \$ 15-27$ i . . . . . . . 1982

II. Hauptstück. Verbandsklage. $\int \mathbb{S} 28-30 \ldots \ldots 13$

III. Hauptstück. Ergänzende Bestimmungen. $\$ \rrbracket 30 a-42 \quad \ldots 2021$

\section{IPR-Gesetz (Neumayr)}

Abschnitt 1. Allgemeine Bestimmungen. $\$ \mathbb{S 1 - 1 1 ~ \ldots . ~ . ~ . ~ . ~ . ~ . ~} 2044$

Abschnitt 2. Personenrecht. $\mathbb{\int} 12-15 \ldots \ldots \ldots 2069$

Abschnitt 3. Familienrecht. $\int \mathbb{S} 16-27 \mathrm{~d} \ldots \ldots \ldots \ldots \ldots . \ldots . \ldots 2072$

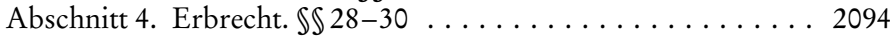

Abschnitt 5. Sachenrecht. $\$ \mathbb{S} 31-33$ a . . . . . . . . . . 2100

Abschnitt 6. Immaterialgüterrechte. $\$ 34 \ldots \ldots \ldots \ldots . \ldots 2106$

Abschnitt 7. Schuldrecht. $\mathbb{\$} \$ 35-49 \ldots \ldots \ldots \ldots . \ldots \ldots 2109$

Abschnitt 8. Schlußbestimmungen. $\int \mathbb{S} 50-54 \ldots \ldots \ldots \ldots 2120$

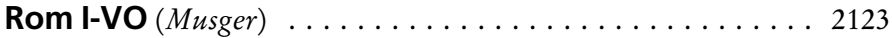

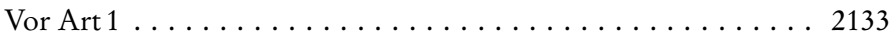

Kapitel I. Anwendungsbereich. Art 1-2 . . . . . . . . . . . 2134 


\section{Inhaltsverzeichnis}

Kapitel II. Einheitliche Kollisionsnormen. Art3-18 . . . . . . . 2140 Kapitel III. Sonstige Vorschriften. Art 19-28 . . . . . . . . . 2173 Kapitel IV. Schlussbestimmungen. Art 29 . . . . . . . . . . 2180

Rom II-VO (Neumayr) $\ldots \ldots \ldots \ldots \ldots \ldots \ldots \ldots \ldots$. . . . . . 2181

Vor Art $1 \ldots \ldots \ldots \ldots \ldots \ldots \ldots . \ldots \ldots 2189$

Kapitel I. Anwendungsbereich. Art 1-3 . . . . . . . . . . . 2192

Kapitel II. Unerlaubte Handlungen. Art 4-9 . . . . . . . . . . 2197

Kapitel III. Ungerechtfertigte Bereicherung, Geschäftsführung ohne Auftrag und Verschulden bei Vertragsverhandlungen.

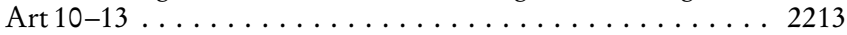

Kapitel IV. Freie Rechtswahl. Art 14 . . . . . . . . . . . . 2220

Kapitel V. Gemeinsame Vorschriften. Art 15-22 . . . . . . . . 2224

Kapitel VI. Sonstige Vorschriften. Art 23-28 . . . . . . . . . . 2233

Kapitel VII. Schlussbestimmungen. Art 29-32 . . . . . . . . 2239

Sachverzeichnis ....................... 2243 


\section{Benutzerhinweise}

Trotz der Konzeption als Kurzkommentar haben wir im Sinne besserer Lesbarkeit im Text weitestgehend auf Abkürzungen verzichtet; alle dennoch verwendeten Abkürzungen, die soweit möglich den $\mathrm{AZR}^{6}$ (2008) folgen, sollten sich im entsprechenden Verzeichnis (S XXXI) finden.

Normtexte ohne Zusatz stammen aus der Stammfassung des betreffenden Gesetzes; Hinweise wie „idF BGBl ..." verweisen auf die jeweils letzte Änderung der Norm. Wird bloß auf „BGBl ...“ hingewiesen, handelt es sich um eine neu eingefügte Bestimmung. Vollzitate von Verordnungen und Richtlinien der EG sind über die im Text verwendeten Kurzzitate im Sachregister aufzufinden.

Bei der Literaturauswahl wurde restriktiv vorgegangen. Vorrang wurde aktuellen Werken eingeräumt; ebenso solchen, die den entsprechenden Problemkreis besonders ausführlich behandeln und/oder auf weiterführende Publikationen verweisen. Vor allem häufig herangezogene Werke werden abgekürzt zitiert; siehe das Verzeichnis SXIX. Publikationen, die bereits in diesem Verzeichnis enthalten sind, werden auch in den Literaturübersichten vor den einzelnen Paragraphen grundsätzlich nur abgekürzt zitiert. Die Auflagenzahl wird nur bei Gefahr von Missverständnissen angegeben; insb weil manche Bände eines Gesamtwerks in unterschiedlichen Auflagen vorliegen oder (bewusst) aus einer Vorauflage zitiert wird. Ebenfalls aus Raumgründen werden für das Lehrbuch Koziol/Welser und die ABGB-Kommentar-Zitate Kurzformen verwendet: K/W steht für Koziol/Welser, K für Klang, R für Rummel und S für Schwimann. Enthält ein Kommentarzitat bloß die Randzahl, ist damit die Kommentierung desselben Paragraphen oder Artikels gemeint.

Bei der Auswahl der Gerichtsentscheidungen wurde ebenfalls vor allem auf Wichtigkeit und Aktualität geachtet. Bei mehrfachen Veröffent- 


\section{Benutzerhinweise}

lichungen wird grundsätzlich nur eine ausführliche Fundstelle zitiert (bevorzugt SZ, dann JBl, EvBl usw); mit Besprechungen versehene Judikaturveröffentlichungen erhielten Vorrang. Sofern Entscheidungen nicht vom OGH stammen, ist das Gericht angeführt. Seit dem Jahre 1990 ergangene Entscheidungen werden zwecks leichterer Auffindbarkeit im RIS mit Geschäftszahl zitiert; aus Platzgründen aber bei jedem Paragraphen mit weniger als 15 Randzahlen nur im Erstzitat. Findet sich bloß eine Geschäftszahl, ist die Entscheidung bis zur Drucklegung unveröffentlicht geblieben. Bei mehrfachen gleichlautenden Aussagen in Entscheidungen („Entscheidungsreihen“) wird öfters die entsprechende RIS-Justiz-Nummer angegeben (zB RS0008985). Paragraphenzitate ohne weitere Angaben beziehen sich immer auf das kommentierte Gesetz. Die Suche nach konkreten Problemen wird durch ein detailliertes Inhaltsverzeichnis (S XI), durch das Sachverzeichnis (S 2243) sowie durch die Angaben in der Kopfzeile erleichtert, die beim ABGB regelmäßig eine Kurzbezeichnung des betreffenden Hauptstücks enthalten. 


\section{Abgekürzt zitierte Literatur}

Aichborn, Lebenspartnerschaften

Aichhorn, Das Recht der Lebenspartnerschaften (2003)

Bearbeiter in Angst, EO

Angst (Hrsg), Kommentar zur Exekutionsordnung2 (2008)

Apathy, Verwendungsanspruch

Apathy, Der Verwendungsanspruch (1988)

Apatby, EKHG

Apathy, Kommentar zum EKHG (1992)

Bearbeiter, BVR I-III, V, VI

Apathy/Iro/Koziol (Hrsg), Österreichisches Bankvertragsrecht ${ }^{2}$ I (2007), II (2008), III (2008), V (2009), VI (2007)

Apatby/Riedler, SR BT

Apathy/Riedler, Schuldrecht. Besonderer Teil ${ }^{4}$ (2010)

Auckenthaler, Zahlung

Auckenthaler, Irrtümliche Zahlung fremder Schulden (1980)

Bearbeiter, BVR I ${ }^{1}, \mathrm{II}^{1}$

Avancini/Iro/Koziol (Hrsg), Österreichisches Bankvertragsrecht ${ }^{1}$ I (1987), II (1993)

Bacher, Ausgleichsansprüche

Bacher, Ausgleichsansprüche zwischen mehreren Sicherern einer fremden Schuld (1994)

Binder, SachenR

M. Binder, Sachenrecht (2003)

Böbler, Mängelrüge

Böbler, Grundwertungen zur Mängelrüge (2000) 


\section{Abgekürzt zitierte Literatur}

Bollenberger, Irrtum

Bollenberger, Irrtum über die Zahlungsunfähigkeit (1995)

Bollenberger, Commodum

Bollenberger, Das stellvertretende Commodum (1999)

Buchegger, Insolvenzrecht I, III

Bartsch/Pollak/Buchegger (Hrsg), Österreichisches Insolvenzrecht, $\mathrm{I}^{4}(2000), \mathrm{III}^{4}(2002)$

Bearbeiter in Burgstaller/Deixler-Hübner, EO

Burgstaller/Deixler-Hübner (Hrsg), Exekutionsordnung, Loseblatt (ab 1999)

Burgstaller/Neumayr, IZVR

Burgstaller/Neumayr (Hrsg), Internationales Zivilverfahrensrecht II, Loseblatt (ab 2001)

F. Bydlinski, Schadensverursachung

F. Bydlinski, Probleme der Schadensverursachung (1964)

F. Bydlinski, Privatautonomie

F. Bydlinski, Privatautonomie und objektive Grundlagen des verpflichtenden Rechtsgeschäftes (1967)

F. Bydlinski, Methodenlehre

F. Bydlinski, Juristische Methodenlehre und Rechtsbegriff ${ }^{2}$ (1991)

F. Bydlinski, System

F. Bydlinski, System und Prinzipien des Privatrechts (1996)

P. Bydlinski, Gestaltungsrechte

P. Bydlinski, Die Übertragung von Gestaltungsrechten (1986)

P. Bydlinski, Bürgschaft

P. Bydlinski, Die Bürgschaft im österreichischen und deutschen Handels-, Gesellschafts- und Wertpapierrecht (1991)

P. Bydlinski, AT

P. Bydlinski, Allgemeiner Teil ${ }^{5}$ (2010)

P. Bydlinski, Kreditbürgschaft

P. Bydlinski, Die Kreditbürgschaft im Spiegel von aktueller Judikatur und Formularpraxis ${ }^{2}$ (2003)

Bydlinski/Bydlinski, Formgebote

P. Bydlinski/F. Bydlinski, Gesetzliche Formgebote für Rechtsgeschäfte auf dem Prüfstand (2001)

Danzl, EKHG

Danzl, Eisenbahn- und Kraftfahrzeughaftpflichtgesetz ${ }^{8}$ (2007)

$\mathrm{XX}$ 


\section{Abgekürzt zitierte Literatur}

Danzl ua, Schmerzengeld

Danzl/Gutierréz-Lobos/Müller, Das Schmerzengeld in medizinischer und juristischer $\operatorname{Sicht}^{9}$ (2009)

Debn, Formnichtige Rechtsgeschäfte

Dehn, Formnichtige Rechtsgeschäfte und ihre Erfüllung (1998)

Deixler-Hübner, Scheidung

Deixler-Hübner, Scheidung, Ehe und Lebensgemeinschaft ${ }^{10}$ (2009)

Dittrich/Tades, ABGB

Dittrich/Tades (Hrsg), Das Allgemeine Bürgerliche Gesetzbuch ${ }^{37}$ (2009)

Dullinger, Aufrechnung

Dullinger, Handbuch der Aufrechnung (1995)

Dullinger, SR AT

Dullinger, Schuldrecht. Allgemeiner Teil ${ }^{4}$ (2010)

Duursma ua, GesR

Duursma/Duursma-Kepplinger/Roth, Handbuch zum Gesellschaftsrecht (2007)

Duursma-Kepplinger, EV

Duursma-Kepplinger, Eigentumsvorbehalt und Mobilienleasing in der Insolvenz (2002)

Eccher, Erbfolge

Eccher, Antizipierte Erbfolge (1980)

Eccher, ErbR

Eccher, Erbrecht ${ }^{4}$ (2010)

Ehrenzweig, System I/1, I/2, II/1, II/2

Armin Ehrenzweig, System des österreichischen allgemeinen Privatrechts², I/1: Allgemeiner Teil (1951), I/2: Das Sachenrecht (1957), II/1: Das Recht der Schuldverhältnisse (1928), II/2: Familien- und Erbrecht (1937)

Eigner, Interzedentenschutz

Eigner, Interzedentenschutz unter besonderer Berücksichtigung der Ehegattenhaftung (2004)

Faber, Gewährleistungsrecht

W. Faber, Handbuch zum neuen Gewährleistungsrecht (2001)

Fasching, ZPR

Fasching, Lehrbuch des österreichischen Zivilprozeßrechts² (1990)

Bearbeiter in Fasching/Konecny, ZPO I-V, Ergbd

Fasching/Konecny (Hrsg), Kommentar zu den Zivilprozessgesetzen ${ }^{2} \mathrm{I}$ (2000), II/1 (2002), II/2 (2003), III (2004), IV/1 (2005), IV/2 (2007), V/1 (2008), Ergänzungsband Zustellgesetze (2008) 


\section{Abgekürzt zitierte Literatur}

Fenyves, Erbenhaftung

Fenyves, Erbenhaftung und Dauerschuldverhältnis (1982)

Ferrari/Hopf, Eherechtsreform

Ferrari/Hopf (Hrsg), Eherechtsreform in Österreich (2000)

Ferrari/Hopf, Reform

Ferrari/Hopf (Hrsg), Reform des Kindschaftsrechts (2001)

Fischer-Czermak, Mobilienleasing

Fischer-Czermak, Mobilienleasing. Rechtsnatur, Gewährleistung und Gefahrtragung (1995)

Frotz, Kreditsicherungsrecht

G. Frotz, Aktuelle Probleme des Kreditsicherungsrechts - GA für den 4. ÖT I/3 (1970)

Fucik ua, Verkehrsunfall VI

Fucik/Hartl/Schlosser (Hrsg), Handbuch des Verkehrsunfalls VI: Zivilrecht (2005)

Gitschthaler/Höllwerth, EheG

Gitschthaler/Höllwerth, Kommentar zum Ehegesetz (2008)

Grillberger, Gütergemeinschaft

Grillberger, Eheliche Gütergemeinschaft (1982)

Gschnitzer/Faistenberger, FamR

Gschnitzer/Faistenberger, Österreichisches Familienrecht ${ }^{2}$ (1979)

Gschnitzer/Faistenberger, ErbR

Gschnitzer/Faistenberger, Österreichisches Erbrecht ${ }^{2}$ (1984)

Gschnitzer ua, SachenR

Gschnitzer/Faistenberger na, Österreichisches Sachenrecht ${ }^{2}$ (1985)

Gschnitzer ua, SR AT

Gschnitzer/Faistenberger ua, Österreichisches Schuldrecht Allgemeiner $\mathrm{Teil}^{2}$ (1985)

Gschnitzer ua, SR BT

Gschnitzer/Faistenberger ua, Österreichisches Schuldrecht Besonderer Teil und Schadenersatz ${ }^{2}$ (1988)

Gschnitzer ua, AT

Gschnitzer/Faistenberger/Barta ua, Allgemeiner Teil des bürgerlichen Rechts $^{2}$ (1992)

Gusenleitner, Ersitzung

Gusenleitner, Ersitzung als allgemeiner Rechtserwerbstatbestand (2004)

Hämmerle/Wünsch, HR I-II ${ }^{4}, \mathrm{III}^{3}$

Hämmerle/Wünsch, Handelsrecht I I (1990), II ${ }^{4}$ (1993), III $^{3}$ (1979) 


\section{Abgekürzt zitierte Literatur}

Harrer/Zitta, Familie

Harrer/Zitta (Hrsg), Familie und Recht (1992)

Hausmann/Vonkilch, WohnR

Hausmann/Vonkilch (Hrsg), Österreichisches Wohnrecht, Loseblatt (ab 2002)

Heller/Berger/Stix, EO

Heller/Berger/Stix, Kommentar zur Exekutionsordnung ${ }^{11}$ (1979)

Hinteregger, FamR

Hinteregger, Familienrecht ${ }^{4}$ (2009)

Holzhammer/Roth, GesR

Holzhammer/Roth, Gesellschaftsrecht ${ }^{2}$ (1997)

Holzner, Ehevermögen

Holzner, Ehevermögen bei Scheidung und bei Tod (1998)

Hopf/Kathrein, EheR

Hopf/Kathrein, Eherecht ${ }^{2}$ (2005)

Iro, Besitzerwerb

Iro, Besitzerwerb durch Gehilfen (1982)

Iro, SachenR

Iro, Sachenrecht ${ }^{4}$ (2010)

Jabornegg, Zurückbehaltungsrecht

Jabornegg, Zurückbehaltungsrecht und Einrede des nicht erfüllten Vertrages (1982)

Bearbeiter in Jabornegg, HGB

Jabornegg (Hrsg), Kommentar zum HGB (1997 mit Ergänzungsheft 1999)

Bearbeiter in Jabornegg/Artmann, UGB I

Jabornegg/Artmann (Hrsg), Kommentar zum UGB I² (2010)

Bearbeiter in Jabornegg/Strasser, AktG I/1, I/2, II, III, IV bzw I ${ }^{5}$

Jabornegg/Strasser (Hrsg), Kommentar zum Aktiengesetz I/14 (2006), $\mathrm{I} / 2^{4}(2004), \mathrm{II}^{5}(2010)$

Jensik, Miteigentum

Jensik, Miteigentum - Wohnungseigentum (1962)

Jud, Erbschaftskauf

B. Jud, Der Erbschaftskauf (1998)

Jud, Schadenersatz

B. Jud, Schadenersatz bei mangelhafter Leistung (2003)

Kandut, Gewährleistungsrecht

Kandut, Das Gewährleistungsrecht beim Kauf (oJ) 


\section{Abgekürzt zitierte Literatur}

Karasek, ÖNORM

Karasek, ÖNORM B 2110² (2009)

Karner/Koziol, Ideeller Schaden

Karner/Koziol, Der Ersatz ideellen Schadens im österreichischen Recht und seine Reform - GA für den 15. ÖJT II/1 (2003)

Karollus, Schutzgesetzverletzung

Karollus, Funktion und Dogmatik der Haftung aus Schutzgesetzverletzung (1992)

Kalss ua, GesR

Kalss/Nowotny/Schaner, Österreichisches Gesellschaftsrecht ${ }^{6}$ (2008)

Kerschner, Irrtumsanfechtung

Kerschner, Irrtumsanfechtung insbesondere beim unentgeltlichen Geschäft (1984)

Kerschner, FamR

Kerschner, Familienrecht ${ }^{4}$ (2010)

Kerschner, DHG

Kerschner, Dienstnehmerhaftpflichtgesetz ${ }^{2}$ (2004)

Kerschner/Bydlinski, Fälle und Lösungen

Kerschner/P. Bydlinski, Fälle und Lösungen zum bürgerlichen Recht für Fortgeschrittene $^{5}$ (2008)

Bearbeiter/K I-VI

Klang/Gschnitzer, Kommentar zum Allgemeinen bürgerlichen Gesetzbuch $^{2}$ I/1 (1964), I/2 (1962), II (1950), III (1952), IV/1 (1968), IV/2 (1978), V (1954), VI (1951), Ergänzungsband (1977)

Bearbeiter $/ \mathrm{K}^{3}$

Fenyves/Kerschner/Vonkilch (Hrsg), 3. Auflage des von Dr. Heinrich Klang begründeten Kommentars zum ABGB: $\$ \$ \$ 44-100$ (2006), \$S137-267 (2008), IS 888-896 (2008), KSchG (2006)

Kletečka, Gewährleistung

Kletečka, Gewährleistung neu. Kommentar zum GewRÄG für Praxis und Ausbildung (2001)

Kodek, Besitzstörung

Kodek, Die Besitzstörung (2002)

Bearbeiter in Konecny/Schubert, InsolvenzG

Konecny/Schubert (Hrsg), Kommentar zu den Insolvenzgesetzen, Loseblatt (ab 1997)

Korinek/Krejci, MRG-HB

Korinek/Krejci (Hrsg), Handbuch zum Mietrechtsgesetz (1985)

XXIV 


\section{Abgekürzt zitierte Literatur}

Kosesnik-Webrle ua, KSchG

Kosesnik-Wehrle/Lehofer/Mayer/Langer, Konsumentenschutzgesetz ${ }^{2}$ (2004)

Koziol, Garantievertrag

Koziol, Der Garantievertrag (1981)

Koziol, HPR I ${ }^{3}$, II ${ }^{2}$

Koziol, Österreichisches Haftpflichtrecht I ${ }^{3}$ (1997), II $^{2}$ (1984)

Koziol, Schadenersatzrecht

Koziol, Grundfragen des Schadenersatzrechts (2010)

$\mathrm{K} / \mathrm{W}$ I, II

Koziol/Welser, Bürgerliches Recht ${ }^{13}$ I (2006) und II (2007), bearbeitet von Kletečka (11. und 12. Auflage bearbeitet von Koziol unter Mitarbeit von Bollenberger) (Bd I) bzw von Welser (Bd II) auf Grundlage des von Koziol und Welser bis zur 10. Auflage gemeinsam verfassten Werkes

Kralik, ErbR

Kralik, Das Erbrecht (1983)

Krejci, KSchG-HB

Krejci (Hrsg), Handbuch zum Konsumentenschutzgesetz (1981)

Krejci, Reform

Krejci, Reform des Gewährleistungsrechts (1994)

Krejci, GesR I, II

Krejci, Gesellschaftsrecht I (2005), II (2010)

Bearbeiter/RK UGB bzw ABGB

Krejci (Hrsg), Kommentar zu den durch das HaRÄG 2005 eingeführten

Neuerungen im Unternehmensgesetzbuch und im Allgemeinen bürgerlichen Gesetzbuch (2007)

Krejci ua, Provisionen

Krejci/Ruppe/Schick, Unerlaubte Provisionen, Zuwendungen und Vorteile im Straf-, Privat- und Steuerrecht (1982)

Kuderna, Entlassungsrecht

Kuderna, Das Entlassungsrecht ${ }^{2}$ (1994)

Kurschel, Gewährleistung

Kurschel, Die Gewährleistung beim Werkvertrag (1989)

Larenz/Canaris, SchuldR II/2 ${ }^{13}$

Larenz/Canaris, Lehrbuch des Schuldrechts: Besonderer Teil II/ $2^{13}$ (1994) [deutsches Recht]

Löschnigg, AR

Löschnigg, Arbeitsrecht ${ }^{11}$ (2009) 


\section{Abgekürzt zitierte Literatur}

Lukas, Zession

Lukas, Zession und Synallagma (2000)

Maultaschl ua, Rechtslexikon

Maultaschl/Schuppich/Stagel (Hrsg), Rechtslexikon - Handbuch des österreichischen Rechts für die Praxis, Loseblatt (ab 1956); zitiert mit dem jeweiligen Schlagwort

Mayrbofer, SR AT

Mayrhofer, Das Recht der Schuldverhältnisse. Allgemeine Lehren (1986)

Meissel, Geschäftsführung

Meissel, Geschäftsführung ohne Auftrag (1993)

Möschl, Lebensgemeinschaft

Möschl, Die nichteheliche Lebensgemeinschaft ${ }^{3}$ (2007)

Bearbeiter/MKBGB

Münchener Kommentar zum BGB in 11 Bänden und einem LoseblattErgänzungsband, erscheint seit 2006 in 5. Auflage [deutsches Recht]

Nademleinsky/Neumayr, IFR

Nademleinsky/Neumayr, Internationales Familienrecht (2007)

Ofner I, II

Ofner, Der Ur-Entwurf und die Berathungs-Protokolle des Österreichischen Allgemeinen bürgerlichen Gesetzbuches, I (1888), II (1889)

Ostheim, Rechtsfähigkeit

Ostheim, Zur Rechtsfähigkeit von Verbänden im österreichischen bürgerlichen Recht (1967)

Ostheim, Familienrechtsreform

Ostheim (Hrsg), Schwerpunkte der Familienrechtsreform 1977/1978 (1979)

Bearbeiter/Palandt

Palandt (Begr), Kurzkommentar zum Bürgerlichen Gesetzbuch ${ }^{69}$ (2010) [deutsches Recht]

Posch, IPR

Posch, Internationales Privatrecht ${ }^{5}$ (2010)

Ch. Rabl, Treuhänder

Ch. Rabl, Der untreue Treuhänder (2002)

Ch. Rabl, Gefahrtragung

Ch. Rabl, Die Gefahrtragung beim Kauf (2002)

Th. Rabl, Bürgschaft

Th. Rabl, Die Bürgschaft (2000) 


\section{Abgekürzt zitierte Literatur}

Rauch-Kallat/Pichler, Entwicklungen

Rauch-Kallat/J. Pichler (Hrsg), Entwicklungen in den Rechten des Kindes im Hinblick auf das UN-Übereinkommen über die Rechte des Kindes (1994)

Bearbeiter in Rechberger, AußStrG

Rechberger (Hrsg), Außerstreitgesetz (2006)

Bearbeiter in Rechberger, ZPO

Rechberger (Hrsg), Kommentar zur $\mathrm{ZPO}^{3}$ (2006)

Rechberger/Kletečka, Bodenrecht

Rechberger/Kletečka (Hrsg), Bodenrecht in Österreich (2004)

Rechberger/Simotta, ZPR

Rechberger/Simotta, Grundriss des österreichischen Zivilprozessrechts ${ }^{7}$ (2009)

Reckenzaun, Bestandgeberpfandrecht

Reckenzaun, Das gesetzliche Bestandgeberpfandrecht (1989)

Bearbeiter, IVR

Reithmann/Martiny, Internationales Vertragsrecht ${ }^{7}$ (2010)

Riedler, Gesamt- und Teilgläubigerschaft

Riedler, Gesamt- und Teilgläubigerschaft im österreichischen Recht (1998)

Riss, Erhaltungspflicht

Riss, Die Erhaltungspflicht des Vermieters (2005)

Bearbeiter/R

Rummel (Hrsg), Kommentar zum Allgemeinen bürgerlichen Gesetzbuch $^{3}$ I (2000 mit Ergänzungsband 2003), II/1 (2002), II/2 a (2007), II/2b (2004), II/3 (2002), II/4 (2002), II/5 (2003), II/6 (2004)

Ruppe, Familienverträge

Ruppe (Hrsg), Handbuch der Familienverträge ${ }^{2}$ (1985)

Samek, Pflichtteilsrecht

Samek, Das österreichische Pflichtteilsrecht samt Anrechnungsrecht (2004)

Schey, Obligationsverhältnisse

Schey, Die Obligationsverhältnisse des österreichischen allgemeinen

Privatrechts I/1, I/2, I/3: I/1: Einleitung - Das Darlehen (1890), I/2:

Der Leihvertrag (1895), I/3: Der Bevollmächtigungsvertrag (1907)

Schragel, AHG

Schragel, Kommentar zum Amtshaftungsgesetz ${ }^{3}$ (2003) 


\section{Abgekürzt zitierte Literatur}

Bearbeiter/S

Schwimann (Hrsg), Praxiskommentar zum Allgemeinen Bürgerlichen Gesetzbuch $^{3}$ I (2005), II (2005), III (2006), IV (2006), V (2006), VI (2006), VII (2005), Ergänzungsband (2007)

Schwimann, IPR

Schwimann, Internationales Privatrecht und Europarecht ${ }^{3}$ (2001)

Schwimann/Kolmasch, Unterhaltsrecht

Schwimann/Kolmasch, Unterhaltsrecht ${ }^{4}$ (2009)

Schwind, EheR

Schwind, Kommentar zum österreichischen Eherecht ${ }^{2}$ (1980)

Schwind, FamR

Schwind, Das Familienrecht (1984)

Spielbüchler, Schuldverhältnis

Spielbüchler, Der Dritte im Schuldverhältnis (1973)

Spielbüchler/Grillberger, AR I

Spielbüchler/Grillberger, Arbeitsrecht I I ${ }^{4}$ Individualarbeitsrecht (1998)

Spitzer, Pfandverwertung

Spitzer, Die Pfandverwertung im Zivil- und Handelsrecht (2004)

Standinger, $\mathrm{BGB}^{13}$ bzw 14

Staudinger (Begr), Kommentar zum bürgerlichen Gesetzbuch mit Einführungsgesetz und Nebengesetzen ${ }^{13}$, erscheint seit 1993 in 13. Auflage, danach (weitergezählte) Bearbeitungen nach Bedarf [deutsches Recht]

Bearbeiter in Straube, HGB I ${ }^{3}$, $\mathrm{II}^{2}$

Straube (Hrsg), Kommentar zum HGB, I ${ }^{3}$ (2003), $\mathrm{II}^{2}$ (2000)

Bearbeiter in Straube, UGB I

Straube (Hrsg), Wiener Kommentar zum Unternehmensgesetzbuch I ${ }^{4}$, Loseblatt (ab 2009)

Umlauft, Anrechnung

Umlauft, Die Anrechnung von Schenkungen und Vorempfängen im Erb- und Pflichtteilsrecht (2001)

Welser, Vertretung

Welser, Vertretung ohne Vollmacht (1970)

Welser, Rat

Welser, Die Haftung für Rat, Auskunft und Gutachten (1983)

Welser, Schadenersatz

Welser, Schadenersatz statt Gewährleistung (1994)

Welser/Jud, Gewährleistung

Welser/B. Jud, Die neue Gewährleistung (2001) 


\section{Abgekürzt zitierte Literatur}

Wilburg, Bereicherung

Wilburg, Die Lehre von der ungerechtfertigten Bereicherung nach österreichischem und deutschem Recht (1934)

Wilburg, Elemente

Wilburg, Die Elemente des Schadensrechts (1941)

Wilhelm, Vertretung

Wilhelm, Die Vertretung der Gebietskörperschaften im Privatrecht (1981)

Worthing-Smith, Unzulässige Klauseln

Worthing-Smith, Unzulässige Klauseln in Mietverträgen (2009)

Würth/Zingher/Kovanyi, Miet- und WohnR

Würth/Zingher/Kovanyi, Miet- und Wohnrecht I ${ }^{22}$ (2009)

Zankl, Vorausvermächtnis

Zankl, Das gesetzliche Vorausvermächtnis des Ehegatten (1996)

Zeiller I-IV

Zeiller, Commentar über das allgemeine bürgerliche Gesetzbuch, I (1811), II (1812), III (1812), IV (1813), Registerband (1813) 


\section{Abkürzungsverzeichnis}

$\mathrm{aA}$

ABB 2000

ABG

ABGB

$\mathrm{AbgEO}$

ABl

abl

Abs

abw

AcP

AdÜ

aE

AEUV

$\mathrm{aF}$

AG

AGB

AGBKr

AHG

AHGB

AHK

AKG

AktG

AKV anderer Ansicht

am angeführten Ort

Allgemeine Bankbedingungen 2000 (von der Bundessektion Bank + Versicherung der Wirtschaftskammer Österreich hrsg Musterbedingungen)

Allgemeines Berggesetz RGBl 1854/146

Allgemeines bürgerliches Gesetzbuch JGS 946

Abgabenexekutionsordnung BGBl 1949/104

Amtsblatt

ablehnend

Absatz

abweichend

Archiv für die civilistische Praxis

Haager Adoptionsübereinkommen BGBl 1978/581

am Ende

Vertrag über die Arbeitsweise der Europäischen

Union ABl 2008 C 115 S 47

alte Fassung

Aktiengesellschaft

Allgemeine Geschäftsbedingungen

Allgemeine Geschäftsbedingungen der österreichischen Kreditunternehmungen, Fassung 1979

Amtshaftungsgesetz BGBl 1949/20

Allgemeines Handelsgesetzbuch RGBl 1863/1

Autonome Honorar-Kriterien

Arbeiterkammergesetz 1992 BGBl 1991/626

Aktiengesetz 1965 BGBl 98

Alpenländischer Kreditorenverband für Kreditschutz und Betriebswirtschaft 


\section{Abkürzungsverzeichnis}

AllgGAG

ALR

Alt

AlVG

aM

AMFG

AMG

AnfO

AngG

Anh

Anm

AnwBl

$\mathrm{AO}$

ApKG

APSG

ARÄG

Arb

ArbVG

ARD

$\arg$

ARGE

Art

ÄrzteG

ASchG

ASFINAG

ASG

ASGG

ASoK

ASVG

AtomHG

ATS

AÜG

AusbV

AusbVorbG ausf

AuslBG

AußStr-BegleitG AußStrG 1854
Allgemeines Grundbuchsanlegungsgesetz BGBl 1930/2

Preußisches Allgemeines Landrecht

Alternative

Arbeitslosenversicherungsgesetz 1977 BGBl 609

(Wv)

anderer Meinung

Arbeitsmarktförderungsgesetz BGBl 1969/31

Arzneimittelgesetz BGBl 1983/185

Anfechtungsordnung RGBl 1914/337

Angestelltengesetz BGBl 1921/292

Anhang

Anmerkung(en)

Österreichisches Anwaltsblatt

Ausgleichsordnung (Wv) BGBl II 1934/221 idF IRÄG 1982 BGBl 370 Art I

Apothekerkammergesetz BGBl 1947/152

Arbeitsplatz-Sicherungsgesetz 1991 BGBl 683

Arbeitsrechtsänderungsgesetz 2000 BGBl I 2000/44

Sammlung arbeitsrechtlicher Entscheidungen

Arbeitsverfassungsgesetz BGBl 1974/22

ARD-Betriebsdienst

argumento (folgt aus)

Arbeitsgemeinschaft

Artikel

Ärztegesetz 1998 BGBl I 1998/169

ArbeitnehmerInnenschutzgesetz BGBl 1994/450

Autobahnen- und Schnellstraßen-Finanzierungs-

Aktiengesellschaft (s BGBl 1982/591)

Arbeits- und Sozialgericht

Arbeits- und Sozialgerichtsgesetz BGBl 1985/104

Arbeits- und Sozialrechtskartei

Allgemeines Sozialversicherungsgesetz BGBl 1955/189

Atomhaftungsgesetz 1999 BGBl I 1998/170

Österreichischer Schilling

Arbeitskräfteüberlassungsgesetz BGBl 1988/196

Ausbeutungsverordnung BGBl 1933/66

Ausbildungsvorbehaltsgesetz BGBl 1996/378 Art II ausführlich

Ausländerbeschäftigungsgesetz BGBl 1975/218

Außerstreit-Begleitgesetz BGBl I 2003/112

Außerstreitgesetz RGBl 1854/208 


\section{Abkürzungsverzeichnis}

AußStrG 2003

AVB

AVG

AVRAG

AZG

AZR

BAG

BAO

BauKG

BauO

BauRG

BauRGNov 1990

BBG

bbl

$\mathrm{Bd}$

BDG

bearb

Begr

begr

BEinstG

Bekl

bekl

BergG

betr

BewG

BG

BGB

BGBl

BGBlG

$\mathrm{BGH}$

BGHZ

B-GlBG

bgld

BinnSchiffG

BK
Außerstreitgesetz BGBl I 2003/111

Allgemeine Versicherungs-Bedingungen

Allgemeines Verwaltungsverfahrensgesetz 1991

BGBl $51(\mathrm{Wv})$

Arbeitsvertragsrechts-Anpassungsgesetz BGBl 1993/459

Arbeitszeitgesetz BGBl 1969/461

Abkürzungs- und Zitierregeln der österreichischen

Rechtssprache und europarechtlicher Rechtsquellen samt Abkürzungsverzeichnis, 6. Auflage 2008, begr von Friedl/Loebenstein, bearb von Dax/Hopf

Berufsausbildungsgesetz BGBl 1969/142

Bundesabgabenordnung BGBl 1961/194

Bauarbeitenkoordinationsgesetz BGBl I 1999/37

Bauordnung

Baurechtsgesetz RGBl 1912/86

Baurechtsgesetznovelle 1990 BGBl 258

Bundesbahngesetz 1992 BGBl 825

Baurechtliche Blätter

Band

Beamten-Dienstrechtsgesetz 1979 BGBl 333

bearbeitet

Begründer

begründet

Behinderteneinstellungsgesetz BGBl 1970/22 idF

BGBl 1988/721

Beklagte(r)

beklagt(e)

Berggesetz 1975 BGBl 259

betreffend

Bewertungsgesetz 1955 BGBl 148

a) Bezirksgericht

b) Bundesgesetz

(deutsches) Bürgerliches Gesetzbuch

Bundesgesetzblatt

Bundesgesetz über das Bundesgesetzblatt 1996

BGBl 1996/660

(deutscher) Bundesgerichtshof

Entscheidungen des (deutschen) Bundesgerichtshofs in Zivilsachen

Bundes-Gleichbehandlungsgesetz BGBl 1993/100

burgenländisch

Binnenschifffahrtsgesetz dRGBl 1898, 868

Bundeskanzler 


\section{Abkürzungsverzeichnis}

B-KUVG

Blg

$\mathrm{BlgHH}$

BlgNR

BM

$\mathrm{BMaA}$

BMF

BMJ

BMSG

BMSVG

BMWA

BörseG

BPG

BPGG

1. BRBG

Bsp

BStFG

BStG

BSVG

BT-Dr

BTVG

BUAG

BV

BVergG

BVG

B-VG

BWG

BZÖ

bzw

$\mathrm{ca}$

CC
Beamten-Kranken- und Unfallversicherungsgesetz

BGBl 1967/200

$\operatorname{Beilage}(\mathrm{n})$

Beilage(n) zu den stenographischen Protokollen des Herrenhauses

Beilage(n) zu den stenographischen Protokollen des Nationalrates

Bundesminister(ium)

Bundesminister(ium) für auswärtige Angelegenheiten

Bundesminister(ium) für Finanzen

Bundesminister(ium) für Justiz

Bundesminister(ium) für soziale Sicherheit, Generationen und Konsumentenschutz

Betriebliches Mitarbeiter- und Selbständigenvorsorgegesetz BGBl I 2002/100

Bundesminister(ium) für wirtschaftliche Angelegenheiten

Börsegesetz 1989 BGBl 555

Betriebspensionsgesetz BGBl 1990/282

Bundespflegegeldgesetz BGBl 1993/110

Erstes Bundesrechtsbereinigungsgesetz BGBl I 1999/191

Beispiel(e)

a) Bundes-Stiftungs- und Fondsgesetz BGBl 1975/11

b) Bundesstraßenfinanzierungsgesetz $1996 \mathrm{BGBl}$ 201

Bundesstraßengesetz 1971 BGBl 286

Bauern-Sozialversicherungsgesetz BGBl 1978/559

Bundestags-Drucksache (deutsch)

Bauträgervertragsgesetz BGBl I 1997/7

Bauarbeiter-Urlaubs- und Abfertigungsgesetz BGBl 1972/414 idF BGBl 1987/618

Besloten Vennootschap (eine der GmbH ähnliche

Gesellschaftsform nach niederländischem Recht)

Bundesvergabegesetz 2002 BGBl I 2002/99

Bundesverfassungsgesetz

Bundes-Verfassungsgesetz BGBl 1930/1 (Wv)

Bankwesengesetz BGBl 1993/532

Bündnis Zukunft Österreich

beziehungsweise

cirka (ungefähr)

(italienischer) Codice civile 
CIC

cic

CIEC

CIM

CIV

CMR

COTIF

D

DaKR ̈̈G

dAnwBl

DAR

DDR

dems

dens

DepG

ders

DevG

DFB

dh

DHG

dies

DMSG

DRdA

dRdA

DREvBl

DRG 2006

dRGBl

DSG

dUrhG

1. DVEheG

E

EA

EAVG

ebd codex iuris canonici

culpa in contrahendo

Commission Internationale de l'Etat Civil (Internationale Kommission für das Zivilstandswesen)

Internationales Übereinkommen über den Eisenbahnfrachtverkehr BGBl 1974/744

Internationales Übereinkommen über den Eisenbahn-Personen- und Gepäckverkehr BGBl 1974/744 Übereinkommen über den Beförderungsvertrag im internationalen Straßengüterverkehr BGBl 1961/138

Übereinkommen über den internationalen Eisenbahnverkehr BGBl 1985/225

Digesten

Darlehens- und Kreditrechts-Änderungsgesetz

BGBl I 2010/28

deutsches Anwaltsblatt

Deutsches Autorecht (Zeitschrift)

Deutsche Demokratische Republik

demselben

denselben

Depotgesetz BGBl 1969/424

derselbe

Devisengesetz BGBl 1946/162

Druckfehlerberichtigung

das heißt

Dienstnehmerhaftpflichtgesetz BGBl 1965/80

dieselbe $(n)$

Denkmalschutzgesetz BGBl 1923/533 idF BGBl I 1999/170

Das Recht der Arbeit

(deutsches) Recht der Arbeit

Evidenzblatt der Rechtsmittelentscheidungen als

Beilage zum deutschen Recht (C)

Deregulierungsgesetz 2006 BGBl I 2006/113

(deutsches) Reichsgesetzblatt

Datenschutzgesetz 2000 BGBl I 1999/165

(deutsches) Gesetz über Urheberrecht und verwandte Schutzrechte dBGBl 1965 I 1273

Erste Durchführungsverordnung zum Ehegesetz dRGBl 1938 I 923

Entscheidung(en)

Einigungsamt

Energieausweis-Vorlage-Gesetz BGBl I 2006/137

ebenda 


\section{Abkürzungsverzeichnis}

\begin{tabular}{|c|c|}
\hline EBG & Eisenbahnbeförderungsgesetz BGBl 1988/180 \\
\hline ECG & E-Commerce-Gesetz BGBl I 2001/152 \\
\hline ecolex & Fachzeitschrift für Wirtschaftsrecht \\
\hline EDV & Elektronische Datenverarbeitung \\
\hline EDVuR & EDV und Recht \\
\hline EEG & Eingetragene Erwerbsgesellschaft \\
\hline $\mathrm{EF}$ & Ehe- und familienrechtliche Entscheidungen \\
\hline $\mathrm{EF}-\mathrm{Z}$ & Zeitschrift für Ehe- und Familienrecht \\
\hline EFZG & Entgeltfortzahlungsgesetz BGBl 1974/399 \\
\hline EG & $\begin{array}{l}\text { a) EGV idF des Amsterdamer Vertrages } \\
\text { b) Europäische Gemeinschaft }\end{array}$ \\
\hline EGAHGB & $\begin{array}{l}\text { Einführungsgesetz zum Allgemeinen Handelsge- } \\
\text { setzbuch RGBl 1863/1 }\end{array}$ \\
\hline EGBGB & $\begin{array}{l}\text { Einführungsgesetz zum (deutschen) Bürgerlichen } \\
\text { Gesetzbuch dRGBl 1896, } 604\end{array}$ \\
\hline EGEO & $\begin{array}{l}\text { Einführungsgesetz zur Exekutionsordnung BGBl } \\
1953 / 6\end{array}$ \\
\hline EGG & Erwerbsgesellschaftengesetz BGBl 1990/257 \\
\hline EGJN & $\begin{array}{l}\text { Einführungsgesetz zur Jurisdiktionsnorm RGBl } \\
1895 / 110\end{array}$ \\
\hline EGMR & Europäischer Gerichtshof für Menschenrechte \\
\hline EGr & Erwägungsgrund \\
\hline EGV & $\begin{array}{l}\text { Vertrag zur Gründung der Europäischen Gemein- } \\
\text { schaften }\end{array}$ \\
\hline EGZPO & $\begin{array}{l}\text { Einführungsgesetz zur Zivilprozeßordnung RGBl } \\
1895 / 112\end{array}$ \\
\hline EheG & Ehegesetz dRGBl I 1938, 807 \\
\hline EheRÄG 1999 & Eherechts-Änderungsgesetz 1999 BGBl I 1999/125 \\
\hline EheRwG & $\begin{array}{l}\text { Bundesgesetz über die Neuordnung der persön- } \\
\text { lichen Rechtswirkungen der Ehe BGBl 1975/412 }\end{array}$ \\
\hline Einl & Einleitung \\
\hline EisbEG & $\begin{array}{l}\text { Eisenbahn-Enteignungsentschädigungsgesetz (frü- } \\
\text { her: Eisenbabnenteigungsgesetz) } 1954 \mathrm{BGBl} 71\end{array}$ \\
\hline EisbKrV & Eisenbahn-Kreuzungsverordnung 1961 BGBl 2 \\
\hline EisenbahnEntG & $\begin{array}{l}\text { Eisenbahnenteignungsgesetz } 1954 \text { BGBl } 2 \text { (nun- } \\
\text { mehr: EisbEG) }\end{array}$ \\
\hline EKEG & Eigenkapitalersatz-Gesetz BGBl I 2003/92 \\
\hline EKHG & $\begin{array}{l}\text { Eisenbahn- und Kraftfahrzeughaftpflichtgesetz } \\
\text { BGBl 1959/48 }\end{array}$ \\
\hline EKZ & Einkaufszentrum, -zentren \\
\hline ElWOG & $\begin{array}{l}\text { Elektrizitätswirtschafts- und -organisationsgesetz } \\
\text { BGBl I 1998/143 }\end{array}$ \\
\hline $\begin{array}{l}\text { EKMR } \\
\text { endg }\end{array}$ & $\begin{array}{l}\text { Europäische Kommission für Menschenrechte } \\
\text { endgültig }\end{array}$ \\
\hline
\end{tabular}




\section{Abkürzungsverzeichnis}

\begin{tabular}{|c|c|}
\hline EntmO & Entmündigungsordnung RGBl 1916/207 \\
\hline $\mathrm{EO}$ & $\begin{array}{l}\text { Exekutionsordnung RGBl 1896/79 idF BGBl } \\
\text { 1995/519 }\end{array}$ \\
\hline $\mathrm{eP}$ & eingetragene Partner \\
\hline EP & eingetragene Partnerschaft(en) \\
\hline EPG & $\begin{array}{l}\text { Eingetragene Partnerschaft-Gesetz BGBl I } \\
2009 / 135\end{array}$ \\
\hline ErbRÄG & Erbrechtsänderungsgesetz 1989 BGBl 656 \\
\hline ErbStG & $\begin{array}{l}\text { Erbschafts- und Schenkungssteuergesetz } 1955 \\
\text { BGBl } 141\end{array}$ \\
\hline Erg & Ergänzung \\
\hline ErgBd & Ergänzungsband \\
\hline ErgLfg & Ergänzungslieferung(en) \\
\hline Erk & Erkenntnis \\
\hline Erl & a) Erläuterung(en) \\
\hline & $\begin{array}{l}\text { b) Erläuterungen zur Regierungsvorlage } \\
\text { c) Erlass }\end{array}$ \\
\hline Erl UGB & Erl 1058 BlgNR 22. GP \\
\hline EStG 1988 & Einkommenssteuergesetz 1988 BGBl 400 \\
\hline EU & Europäische Union \\
\hline EuBewVO & $\begin{array}{l}\text { Verordnung 1206/2001/EG über die Zusammenar- } \\
\text { beit zwischen den Gerichten der Mitgliedstaaten auf } \\
\text { dem Gebiet der Beweisaufnahme in Zivil- oder Han- } \\
\text { delssachen, ABl } 2001 \text { L } 174 \text { S } 1\end{array}$ \\
\hline EuFrÜb & $\begin{array}{l}\text { Europäisches Übereinkommen über die Berechnung } \\
\text { von Fristen BGB1 1983/254 }\end{array}$ \\
\hline EuGEI & Europäisches Gericht erster Instanz \\
\hline EuGH & Europäischer Gerichtshof \\
\hline EuGRZ & Europäische Grundrechte Zeitschrift \\
\hline EuGVÜ & $\begin{array}{l}\text { Europäisches Übereinkommen vom 27.9.1968 über } \\
\text { die gerichtliche Zuständigkeit und die Vollstreckung } \\
\text { gerichtlicher Entscheidungen in Zivil- und Handels- } \\
\text { sachen BGBl III 1998/209 }\end{array}$ \\
\hline EuGVVO & $\begin{array}{l}\text { Verordnung 44/2001/EG über die gerichtliche Zu- } \\
\text { ständigkeit und Vollstreckung von Entscheidungen } \\
\text { in Zivil- und Handelssachen, ABl } 2001 \mathrm{~L} 12 \mathrm{~S} 1\end{array}$ \\
\hline EuInsVO & $\begin{array}{l}\text { Verordnung 1346/2000/EG über Insolvenzverfah- } \\
\text { ren, ABl } 2000 \text { L } 160 \text { S } 1\end{array}$ \\
\hline EujurZ & Eurojuris-Zeitung \\
\hline EURIBOR & Euro Interbank Offered Rate \\
\hline 1. Euro-JuBeG & 1. Euro-Justiz-Begleitgesetz BGBl I 1998/125 \\
\hline EUV & $\begin{array}{l}\text { Vertrag über die Europäische Union ABl } 1992 \text { C } 191 \\
\text { S } 1\end{array}$ \\
\hline EUVerf & Europäische Verfassung \\
\hline
\end{tabular}




\section{Abkürzungsverzeichnis}

EuUVO

EuVTVO

EuZ

EuZA

EuZW

EV

$\mathrm{eV}$

EvBl

4. EVHGB

EVÜ

EWG

EWIV

EWIVG

EWr

EWR

EWS

f, ff

FamRAnglV

FamErbR ̈̈G

FamLAG

FamRZ

FamZ

FBG

FernFinG

FG

FinSG

FinStrG

FIS
Verordnung 4/2009/EG über die Zuständigkeit, das anwendbare Recht, die Anerkennung und Vollstreckung von Entscheidungen und die Zusammenarbeit in Unterhaltssachen, ABl 2009 L 7 S 1

Verordnung 806/2004/EG zur Einführung eines europäischen Vollstreckungstitels für unbestrittene Forderungen, ABl 2004 L 143 S 15

(Schweizer) Zeitschrift für Europarecht

Europäische Zeitschrift für Arbeitsrecht

Europäische Zeitschrift für Wirtschaftsrecht

a) Eigentumsvorbehalt

b) Einführungsverordnung

c) einstweilige Verfügung

eingetragener Verein

Evidenzblatt der Rechtsmittelentscheidungen in

Österreichische Juristen-Zeitung

Vierte Verordnung zur Einführung handelsrecht-

licher Vorschriften im Lande Österreich dRGBl I 1938, 1999

Übereinkommen über das auf vertragliche Schuldverhältnisse anzuwendende Recht BGBl III 1998/208

Europäische Wirtschaftsgemeinschaft

Europäische wirtschaftliche Interessenvereinigung

EWIV-Ausführungsgesetz BGBl 1995/521

Entscheidungen Wohnrecht

Europäischer Wirtschaftsraum

Europäisches Wirtschafts- und Steuerrecht und der, die folgende(n)

Familienrechts-Angleichungsverordnung dRGBl I 1943, 80

Familien- und Erbrechts-Änderungsgesetz 2004

BGBl I 2004/58

Familienlastenausgleichsgesetz 1967 BGB1 376

(deutsche) Zeitschrift für das gesamte Familienrecht Interdisziplinäre Zeitschrift für Familienrecht ( $\mathrm{ab}$ Heft 3/2007: iFamZ)

Firmenbuchgesetz BGBl 1991/10

Fern-Finanzdienstleistungs-Gesetz BGBl I 2004/62

Festgabe

Finanzsicherheiten-Gesetz BGBl I 2003/117

Finanzstrafgesetz BGBl 1958/129

Fédération Internationale de Ski (Internationaler

Skiverband) 


\section{Abkürzungsverzeichnis}

FLAG

FlVfGG

FMA

FMABG

FMedG

FN

FOG

FrG

FS

FTFG

F-VG

G

GA

GAngG

GBG

GBIÖ

GedS

GEG

GenG

GenRevG

Geo

GeS

GesRÄG 2005

GesBR

GeSchG

GesRZ

GewO

GewRÄG

GGG

GH

GKG

GIBG

GIU
Familienlastenausgleichsgesetz 1967 BGBl 376

Flurverfassungs-Grundsatzgesetz 1951 BGBl 103

Finanzmarktaufsicht

Finanzmarktaufsichtsbehördengesetz BGBl I 2001/97

Fortpflanzungsmedizingesetz BGBl 1992/275

Fußnote

Forschungsorganisationsgesetz BGBl 1981/341

Fremdengesetz 1997 BGBl I 1997/75

Festschrift

Forschungs- und Technologieförderungsgesetz

BGBl 1982/434

Finanz-Verfassungsgesetz 1948 BGBl 45 idF BGBl 1996/201 Art 65

Gesetz

Gutachten

Gutsangestelltengesetz BGBl 1923/538

Allgemeines Grundbuchsgesetz 1955 BGBl 39

Gesetzblatt für das Land Osterreich (1938-1940)

Gedenkschrift

Gerichtliches Einbringungsgesetz 1962 BGBl 288

Genossenschaftsgesetz RGBl 1873/70

Genossenschaftsrevisionsgesetz 1997 BGBl I 1997/127

Geschäftsordnung für die Gerichte I. und II. Instanz BGBl 1951/264

Zeitschrift für Gesellschafts- und Steuerrecht

Gesellschaftsrechtsänderungsgesetz 2005 BGBl I 2005/59

Gesellschaft bürgerlichen Rechts

Bundesgesetz zum Schutz vor Gewalt in der Familie BGBl 1996/759

Der Gesellschafter

Gewerbeordnung 1994 BGBl 194 (Wv)

Gewährleistungsrechts-Änderungsgesetz BGBl I 2001/48

Gerichtsgebührengesetz BGBl 1984/501

Die Gerichtshalle

Gerichtskommissärgesetz BGBl 1970/343

Gleichbehandlungsgesetz BGBl 1979/108

Sammlung von zivilrechtlichen Entscheidungen des

k.k. Obersten Gerichtshofes, hrsg von Glaser und Unger 


\section{Abkürzungsverzeichnis}

GIUNF

$\mathrm{GmbH}$

GmbHG

GMG

GoA

GOG

GoldKlG

GP

GPR

GrStG

GRURInt

GSGG

GSpG

GSVG

GTG

GUG

GWG

GZ

$\mathrm{H}$

hA

HaRÄG

HAVE/REAS

$\mathrm{HbG}$

HebG

HeimAufG

$\mathrm{HfD}$

HfKD

HG

HGB

$\mathrm{HHB}$

$\mathrm{hL}$

hM

Hrsg

hrsg

hRspr

HS
Sammlung von zivilrechtlichen Entscheidungen des

k.k. Obersten Gerichtshofes, Neue Folge

Gesellschaft mit beschränkter Haftung

Gesetz über Gesellschaften mit beschränkter

Haftung RGBl 1906/58

Gebrauchsmustergesetz BGBl 1994/211

Geschäftsführung ohne Auftrag

Gerichtsorganisationsgesetz RGBl 1896/217

Goldklauselgesetz BGBl 1937/130

Gesetzgebungsperiode

(deutsche) Zeitschrift für Gemeinschaftsprivatrecht

Grundsteuergesetz 1955 BGBl 149

Gewerblicher Rechtsschutz und Urheberrecht, Internationaler Teil

Güter- und Seilwege-Grundsatzgesetz 1967

BGBl 198

Glückspielgesetz BGBl 1989/620

Gewerbliches Sozialversicherungsgesetz

BGBl 1978/560

Gentechnikgesetz BGBl 1994/510

Grundbuchumstellungsgesetz BGBl 1980/550

Gaswirtschaftsgesetz BGBl I 2000/121

a) Geschäftszahl

b) Österreichische Allgemeine Gerichtszeitung

Heft

herrschende Ansicht

Handelsrechts-Änderungsgesetz

Haftung und Versicherung (Schweizer Zeitschrift)

Hausbesorgergesetz BGBl 1970/16

Hebammengesetz 1963 BGBl 1964/3

Heimaufenthaltsgesetz BGBl I 2004/11

Hofdekret

Hofkanzleidekret

Handelsgericht

Handelsgesetzbuch dRGBl 1897, 219

Herrenhausbericht zur dritten Teilnovelle (s bei Mat III. TN)

herrschende Lehre

herrschende Meinung

Herausgeber

herausgegeben

herrschende Rechtsprechung

a) Handelsrechtliche Entscheidungen

b) Halbsatz 


\section{Abkürzungsverzeichnis}

$\begin{array}{ll}\text { HUP } & \text { Haager Unterhaltsprotokoll vom 23.11.2007 } \\ \text { HVertrG } & \text { Handelsvertretergesetz BGBl 1993/88 } \\ \text { HypBG } & \text { Hypothekenbankgesetz dRGBl 1899,375 } \\ \text { IA } & \text { Initiativantrag } \\ \text { idF } & \text { in der Fassung } \\ \text { idgF } & \text { in der geltenden Fassung } \\ \text { idR } & \text { in der Regel } \\ \text { idS } & \text { in dem, in diesem Sinne } \\ \text { IEG } & \text { Insolvenzrechtseinführungsgesetz BGBl I 1997/114 } \\ & \text { Art III } \\ \text { ieS } & \text { im engeren Sinn } \\ \text { IESG } & \text { Insolvenz-Entgeltsicherungsgesetz BGBl 1977/324 } \\ \text { iFamZ } & \text { Interdisziplinäre Zeitschrift für Familienrecht (bis } \\ & \text { Heft 2/2007: FamZ) } \\ \text { IHR } & \text { Internationales Handelsrecht - Zeitschrift für das } \\ & \text { Recht des internationalen Warenkaufs und -vertriebs } \\ \text { iHv } & \text { in Höhe von } \\ \text { ImmMV } & \text { Verordnung des Bundesministers für wirtschaftliche } \\ & \text { Angelegenheiten über Standes- und Ausübungsre- } \\ \text { ImmoInvFG } & \text { geln für Immobilienmakler BGBl 1996/297 } \\ \text { Immobilien-Investmentfondsgesetz BGBl I } \\ \text { immolex } & \text { 2003/80 } \\ \text { ImmZ } & \text { Neues Miet- und Wohnrecht } \\ \text { infas } & \text { Österreichische Immobilien-Zeitung } \\ \text { insb } & \text { Informationen aus dem Arbeits- und Sozialrecht } \\ \text { InvFG } & \text { insbesondere } \\ \text { IPR } & \text { Investmentfondsgesetz BGBl 1993/532 } \\ \text { IPRax } & \text { Internationales Privatrecht } \\ & \text { Praxis des Internationalen Privat- und Verfahrens- } \\ \text { IPRE } & \text { rechts (deutsche Zeitschrift) } \\ \text { IPRG } & \text { Österreichische Entscheidungen zum internationa- } \\ & \text { len Privatrecht- und Verfahrensrecht } \\ \text { IO } & \text { Bundesgesetz über das internationale Privatrecht } \\ \text { IRÄ-BG } & \text { BGBl 1978/304 } \\ & \text { Insolvenzordnung BGBl I 2010/29 } \\ \text { IRÄG } & \text { Insolvenzrechtsänderungs-Begleitgesetz BGBl I } \\ \text { iSd } & \text { 2010/58 } \\ \text { iSv } & \text { Insolvenzrechtsänderungsgesetz } \\ \text { IT } & \text { im Sinne des, der } \\ \text { iVm } & \text { im Sinne von } \\ \text { IVVG } & \text { Informationstechnik, -technologie } \\ & \text { in Verbindung mit } \\ & \text { Europäischen Wirtschaftsraum BGBl 1993/89 } \\ & \end{array}$




\section{Abkürzungsverzeichnis}

iwS

IZVR

JA

JAB

$\mathrm{JABl}$

JAP

$\mathrm{Jb}$

JB

$\mathrm{JBl}$

JEV

JGS

JherJB

JMV

JMVBl

JMZ

JN

JRP

JUS

jusIT

JWG

JZ

$\mathrm{K}$

$\mathrm{KAKuG}$

KartG

KartG 2005

KautSchG

$\mathrm{KBB}$

KBGG

KEG

KFG

Kfl-Bef Bed

$\mathrm{Kfz}$

KG

KGG

KHVG im weiteren Sinn

Internationales Zivilverfahrensrecht

Justizausschuss

Justizausschussbericht

Amtsblatt der österreichischen Justizverwaltung

Juristische Ausbildung und Praxisvorbereitung

Jahrbuch

Judikatenbuch des Obersten Gerichtshofes

Juristische Blätter

Journal für Erbrecht und Vermögensnachfolge

Justizgesetzsammlung, Gesetze und Verordnungen im Justizfach

Jherings Jahrbücher für Dogmatik des bürgerlichen Rechts

Justizministerialverordnung

Verordnungsblatt des k.k. Justizministeriums

Justizministerialzahl

Jurisdiktionsnorm RGBl 1895/111

Journal für Rechtspolitik

Jus-Extra, Beilage zur Wiener Zeitung

Fachzeitschrift für Rechtsinformation, Datenschutz und IT-Recht

Jugendwohlfahrtsgesetz 1989 BGBl 161

(deutsche) Juristenzeitung

a) Klang-Kommentar (s S XXIV)

b) Kundmachung

Kranken- und Kuranstaltengesetz BGBl 1957/1

(früber: $K A G$ )

Kartellgesetz 1988 BGBl 600

Kartellgesetz 2005 BGBl I 2005/61

Kautionsschutzgesetz BGBl 1937/229

Koziol/Bydlinski/Bollenberger

Kinderbetreuungsgeldgesetz BGBl I 2001/103

a) Kommandit-Erwerbsgesellschaft

b) Kraftloserklärungsgesetz 1951 BGBl 86

Kraftfahrgesetz 1967 BGBl 267

Allgemeine Beförderungsbedingungen für den

Kraftfahrlinienverkehr BGBl II 2001/47

Kraftfahrzeug(e)

a) Kommanditgesellschaft

b) (ehemaliges) Kreisgericht

Karenzgeldgesetz BGBl I 1997/47 Art 1

Kraftfahrzeug-Haftpflichtversicherungsgesetz 1994

BGBl 651 


\section{Abkürzungsverzeichnis}

KindG

KindRÄG 2001

KJBG

KlGG

KMG

$\mathrm{KO}$

KOG

$\mathrm{KOM}$

$\mathrm{KdmPat}$

KRES

krit

Krnt

krnt

KSchG

KSÜ

KUG

$\mathrm{K} / \mathrm{W}$

KWG

$\mathrm{L}$

LAG

LBG

leg cit

LegÜ

LFG

LG

LGBl

LGZ

LiegTeilG

lit

Lit

LKW

LPG

LS

LSK

LuftVG

MarkSchG

Mat
Bundesgesetz über die Neuordnung des Kindschaftsrechts BGBl 1977/403

Kindschaftsrecht-Änderungsgesetz 2001 BGBl I 2000/135

Gesetz über die Beschäftigung von Kindern und Jugendlichen 1987 BGBl 599 (Wv)

Kleingartengesetz BGBl 1959/6

Kapitalmarktgesetz BGBl 1991/625

Konkursordnung RGBl 1914/337 idF IRÄG 1982

BGBl 370 Art II

Kartellobergericht

Dokumente der Kommission der Europäischen

Gemeinschaften

Kundmachungspatent

Konsumentenrecht - Entscheidungssammlung

kritisch

Kärnten, Kärntner

kärntnerisch

Konsumentenschutzgesetz BGBl 1979/140

Haager Kinderschutzübereinkommen

Karenzurlaubsgeldgesetz BGBl 1974/395

Koziol/Welser (s S XXV)

Kreditwesengesetz 1979 BGBl 63

Lehre

Landarbeitsgesetz 1984 BGBl 287 (Wv)

Liegenschaftsbewertungsgesetz BGBl 1992/150

legis citatae (der zitierten Vorschrift)

CIEC-Legitimationsübereinkommen BGBl

1976/102

Luftfahrtgesetz BGBl 1957/253

a) Landesgericht

b) Landesgesetz

Landesgesetzblatt

Landesgericht für Zivilrechtssachen

Liegenschaftsteilungsgesetz BGBl 1930/3

litera (Buchstabe)

Literatur

Lastkraftwagen

Landpachtgesetz BGBl 1969/451

Leitsatz, -sätze

Leitsatzkartei

Luftverkehrsgesetz dRGBl I 1936, 653

Markenschutzgesetz 1970 BGBl 260 (Wv)

Materialien 


\section{Abkürzungsverzeichnis}

Mat III. TN

$\mathrm{mE}$

MedG

MEG

MEntw

Miet

MiFID

migraLex

MinroG

$\mathrm{Mj}$

$\mathrm{mj}$

MMR

MR

MRA

MRG

MR-Int

MRK

MSA

Mschr

MuSchG

MuttSchG

mwN

$\mathrm{NÄG}$

NahVersG

NamRÄG

NBG

$\mathrm{nF}$

NJW

NN

$\mathrm{NO}$

nö

NotAktsG

$\mathrm{Nr}$

NRsp
Kaiserliche Verordnung vom 19. März 1916, RGBl 69, über die dritte Teilnovelle zum ABGB. Mit Materialien (1916; daraus: Herrenhausbericht, S $123 \mathrm{ff}$, zit auch: HHB; dieser auch veröffentlicht als 78 BlgHH, 21. Sess 1912)

meines Erachtens

Mediengesetz BGBl 1981/314

Maß- und Eichgesetz BGBl 1950/152

Ministerialentwurf

Mietrechtliche Entscheidungen

Markets in Financial Instruments Directive (Richtlinie 2004/39/EG über Märkte für Finanzinstrumente)

Zeitschrift für Fremden- und Minderheitenrecht

Mineralrohstoffgesetz BGBl I 1999/38

Minderjährige(r)

minderjährig

MultiMedia und Recht

Medien und Recht

Medien und Recht, Archiv

Mietrechtsgesetz BGBl 1981/520

Medien und Recht International

Europäische Menschenrechtskonvention BGBl

1958/210

Haager Minderjährigenschutzübereinkommen

BGBl 1975/446

Monatsschrift

Musterschutzgesetz 1990 BGBl 497

Mutterschutzgesetz 1979 BGBl 221 (Wv)

mit weiteren Nachweisen

Namensänderungsgesetz BGBl 1988/195

BG zur Verbesserung der Nahversorgung und der

Wettbewerbsbedingungen BGBl 1977/392

Namensrechtsänderungsgesetz BGBl 1995/25

Nationalbankgesetz 1984 BGBl 50 (Wv)

neue Fassung

(deutsche) Neue Juristische Wochenschrift

nomen nescio (unbekannter Autor)

Notariatsordnung RGBl 1871/75

niederösterreichisch

Notariatsaktsgesetz RGBl 1871/76

Nummer

Neue Rechtsprechung des OGH in Österreichische Juristen-Zeitung 


NTG
Nw
NWG
NZ
NZ-K
N\&V
ÖA
ö̈
ÖAKR
ÖAMTC-LSK

ÖBA

ÖBB

OBDK

ÖBl

OEG

$\mathrm{OeNB}$

OG

$\mathrm{OGH}$

OGHG

ÖGIZIN

ÖGZ

$\mathrm{OHG}$

ÖIAG-Gesetz

Notariatstarifgesetz BGBl 1973/576

Nachweise

Notwegegesetz RGBl 1896/140

Österreichische Notariats-Zeitung

Kartei in Außerstreitsachen in Österreichische

Notariats-Zeitung

Nova \& Varia (Zeitschrift des Juristenverbandes)

Der Österreichische Amtsvormund oder Ähnliche(s)

Österreichisches Archiv für Kirchenrecht

Leitsatzkartei des Österreichischen Automobil-,

Motorrad- und Touring Clubs

Österreichisches Bankarchiv

Österreichische Bundesbahnen

Oberste Berufungs- und Disziplinarkommission für Rechtsanwälte und Rechtsanwaltsanwärter

Österreichische Blätter für gewerblichen Rechtsschutz und Urheberrecht

Offene Erwerbsgesellschaft

Oesterreichische Nationalbank

Offene Gesellschaft

Oberster Gerichtshof

Bundesgesetz über den Obersten Gerichtshof BGBl $1968 / 328$

Österreichische Gesellschaft für Information und

Zusammenarbeit im Notariat

Österreichische Gemeinde-Zeitung

Offene Handelsgesellschaft

BG über die Neuordnung der Rechtsverhältnisse der

Österreichischen Industrieholding Aktiengesellschaft und der Post und Telekombeteiligungsverwaltungsgesellschaft BGBl I 2000/24
of
OJT
ÖJZ
ÖJZ-MRK

OLG

oÖ

OPG

OP-Listen

OR ohne Jahr

Österreichischer Juristentag

Österreichische Juristen-Zeitung

MRK-Entscheidungen in Österreichische Juristen-

Zeitung

Oberlandesgericht

oberösterreichisch

Offene Personengesellschaft

offene Posten-Listen

(Schweizer) Obligationenrecht

ORF-G

ORF-Gesetz BGBl I 2001/83

OrgHG

Organhaftpflichtgesetz BGBl 1967/181 


\section{Abkürzungsverzeichnis}

öS

ÖSGRUM

ÖStA

ÖStZ

ÖStZB

ÖZVV

ÖZW

$\mathrm{pa}$

PACS

PartG

PaßG

PatAnwG

PatG

PatVG

PersFrSchG

PfBrG

PG

PHG

PKG

Pkt

PKW

PO

PostG

PreisG

PSG

PStG

PStV

PVInfo

pVV

QuHGZ

$\mathrm{R}$

RabelsZ

RAO

RAT
Österreichischer Schilling

Österreichische Schriftenreihe zum gewerblichen

Rechtsschutz, Urheber- und Medienrecht

Österreichisches Standesamt

Österreichische Steuer-Zeitung

Die finanzrechtlichen Erkenntnisse des $\mathrm{VwGH}$ und des VfGH, Blg zur Österreichischen Steuerzeitung

Österreichisches Zentrales Vertretungsverzeichnis

Österreichische Zeitschrift für Wirtschaftsrecht pro anno, per annum

Pacte civil de solidarité (ziviler Solidaritätspakt; zivilrechtliche Partnerschaft von Paaren unabhängig von deren sexueller Orientierung im französischen Recht)

Parteiengesetz BGBl 1975/404 idF BGBl I 1997/130 Art 13

Paßgesetz 1992 BGBl 839

Patentanwaltsgesetz BGBl 1967/214

Patentgesetz 1970 BGBl 259 (Wv)

Patientenverfügungs-Gesetz BGBl I 2006/55

Bundesverfassungsgesetz zum Schutz der persönlichen Freiheit BGBl 1988/684

Pfandbriefgesetz dRGBl I 1927, 492

Pensionsgesetz 1965 BGBl 340

Produkthaftungsgesetz BGBl 1988/99

Pensionskassengesetz BGBl 1990/281

Punkt

Personenkraftwagen

Postordnung BGBl 1957/110

Postgesetz 1997 BGBl I 1998/18

Preisgesetz 1992 BGBl 145

Privatstiftungsgesetz BGBl 1993/694

Personenstandsgesetz BGBl 1983/60

Personenstandsverordnung BGBl 1983/629

Fachzeitschrift für Personalverrechnung positive Vertragsverletzung

Quartalshefte der Girozentrale

Rummel-Kommentar (s S XXVII)

Rabels Zeitschrift für ausländisches und internationales Privatrecht

Rechtsanwaltsordnung RGBl 1868/96

Bundesgesetz über den Rechtsanwaltstarif

BGBl 1969/189 
RatG

RStDG

$\operatorname{RdM}$

RdS

$\mathrm{RdU}$

RdW

RelKEG

RFG

RGBl

RGZ

RHPflG

RichtWG

RIS

RIW

RK

RL

RL-BA

RohrlG

RPA

RPflG

RpflG

RPflA

Rs

Rsp

Rspr

RStDG

RStG

$\mathrm{RV}$

RZ

$\mathrm{Rz}$

RZ-EÜ

Ratengesetz BGBl 1961/279

Richter- und Staatsanwältedienstgesetz BGBl

1961/305

Recht der Medizin

Recht der Schule

Recht der Umwelt

Österreichisches Recht der Wirtschaft

Gesetz über die religiöse Kindererziehung BGB1 1985/155 (Wv)

Rechts- und Finanzierungspraxis der Gemeinden

Reichsgesetzblatt

Entscheidungen des (deutschen) Reichsgerichts in

Zivilsachen

Reichshaftpflichtgesetz dRGBl 1871, 207

Richtwertgesetz BGBl 1993/800 Art IX

Rechtsinformationssystem des Bundes

Recht der internationalen Wirtschaft

Reformkommentar $=$ Krejci (Hrsg), Kommentar zu den durch das HaRÄG 2005 eingeführten Neuerungen im Unternehmensgesetzbuch und im Allgemeinen bürgerlichen Gesetzbuch (2007)

Richtlinie der EU

Richtlinien für die Ausübung des Rechtsanwaltsberufes, für die Überwachung der Pflichten des

Rechtsanwaltes und für die Ausbildung der Rechtsanwaltsanwärter, kundgemacht im Amtsblatt zur

Wiener Zeitung vom 14.12.1977

Rohrleitungsgesetz BGBl 1975/411

Recht und Praxis der öffentlichen Auftrags-

vergabe

Entscheidungssammlung Grundbuchssachen

Rechtspflegergesetz BGBl 1985/560

Sammelmappe für Rechtspfleger-Besprechungen

Rechtssache (bei Europäischen Gerichten)

Rechtsprechung (Zeitschrift)

Rechtsprechung

Richter- und Staatsanwaltschaftsdienstgesetz BGBl

1961/305 idF BGBl I 2007/96

Rückstellungsgesetz(e)

Regierungsvorlage

Österreichische Richterzeitung

Randzahl

Österreichische Richterzeitung Entscheidungsübersicht 


\section{Abkürzungsverzeichnis}

S

S

$\mathrm{sbg}$

SchBeihG

SchG

SchiedsRÄG

SchOG

SchSpG

SchUG

schw

SchZG

SE

SEG

Sess

SigG

Slg

SMG

$\operatorname{sog}$

SpG

SPG

SpR

SpuRt

SSt

SSV-NF

StadtErnG

StAZ

StbG

StEG 1969

StEG 2005

StGB

StGBl

StGG

Stmk a) Satz

b) Schwimann-Kommentar (s S XXVIII)

c) Seite siehe salzburgerisch

Schülerbeihilfengesetz BGBl 1983/455 (Wv)

Scheckgesetz 1955 BGBl 50

Schiedsrechts-Änderungsgesetz 2006 BGBl I 2006 2006/7

Schulorganisationsgesetz BGBl 1962/242

Schauspielergesetz BGBl 1922/441

Schulunterrichtsgesetz BGBl 1986/472 (Wv)

schweizerisch

Schutzzertifikatsgesetz 1996 BGBl I 1997/11

Societas Europaea (Europäische Gesellschaft)

SE-Gesetz BGBl I 2004/67

Session

Signaturgesetz BGBl I 1999/190

a) Sammlung

b) Sammlung der Rechtsprechung des EuGH und des EuGEI

Suchtmittelgesetz BGBl I 1997/112

so genannte, -er, -es

Sparkassengesetz BGBl 1979/64

Sicherheitspolizeigesetz BGBl 1991/566

Spruchrepertorium des Obersten Gerichtshofes

(deutsche) Zeitschrift für Sport und Recht

Entscheidungen des österreichischen Obersten

Gerichtshofes in Strafsachen und Disziplinarangelegenheiten

Entscheidungen des Obersten Gerichtshofes in

Sozialrechtssachen

Stadterneuerungsgesetz BGBl 1974/287

Das Standesamt (deutsch)

Staatsbürgerschaftsgesetz 1985 BGBl 311 (Wv)

Strafrechtliches Entschädigungsgesetz BGBl $1969 / 270$

Strafrechtliches Entschädigungsgesetz 2005 BGBl I 2005/25

Strafgesetzbuch BGBl 1974/60

Staatsgesetzblatt für die Republik Österreich

Staatsgrundgesetz über die allgemeinen Rechte der

Staatsbürger RGBl 1867/142

Steiermark 


\section{Abkürzungsverzeichnis}

stmk

StPO

str

StRÄG

StrSchG

stRspr

StudFG

StVO

StVÜ

sublit

SV

SVR

SVSlg

SWRÄG 2006

SZ

TabMG

TÄG

taxlex

taxlex-SRa

TEG

TestÜ

Tir

Tir BauO

Tir WWSGG

TKG

TN

TNG

TP

TranspR

TSchG

TVG

ua

$u \ddot{\mathrm{A}}$

uam

UbG

ÜbG

Übk

UFG steiermärkisch

Strafprozessordnung 1975 BGBl 631 (Wv)

streitig

Strafrechtsänderungsgesetz

Strahlenschutzgesetz BGBl 1969/227

ständige Rechtsprechung

Studienförderungsgesetz 1992 BGBl 305

Straßenverkehrsordnung 1960 BGBl 159

Haager Straßenverkehrsübereinkommen

BGBl 1975/387

sublitera (Unterbuchstabe)

Der Sachverständige

Straßenverkehrsrecht (deutsche Zeitschrift)

Sozialversicherungsrechtliche Entscheidungen

Sachwalterrechts-Änderungsgesetz 2006 BGBl I 2006/92

Entscheidungen des österreichischen Obersten

Gerichtshofes in Zivil- (und Justizverwaltungs-)

sachen

Tabakmonopolgesetz 1996 BGBl 1995/830

Tierärztegesetz BGBl 1975/16

Zeitschrift für Steuer und Beratung

taxlex-Steuerradar

Todeserklärungsgesetz 1950 BGBl 1951/23

Haager Testamentsübereinkommen BGBl 1963/295

Tirol, Tiroler

Tiroler Bauordnung LGBl 2001/94

Tiroler Wald- und Weideservitutengesetz

Telekommunikationsgesetz 2003 BGBl I 2003/70

Teilnovelle

Teilzeitnutzungsgesetz BGBl I 1997/32

Tarifpost

Transportrecht (deutsche Zeitschrift)

Tierschutzgesetz BGBl I 2004/118

Tierversuchsgesetz 1988 BGBl 1989/501 idF BGBl I 1999/169

a) und andere(s)

b) unter anderem

und Ähnliche(s)

und andere(s) mehr

Unterbringungsgesetz BGBl 1990/155

Übernahmegesetz BGBl I 1998/127

Übereinkommen

Umweltförderungsgesetz BGBl 1993/185 


\section{Abkürzungsverzeichnis}

UFS

UG 2002

UGB

UHG

UNCITRAL

UN-K

unstr

unzutr

URÄG 2008

UrhG

UrlG

USchG

USt

UStG

USt $\ddot{U}$

usw

$\mathrm{uU}$

uva

UVG

UVS

UWG

V

va

VAG

VBG

VBKG

VbVG

VEG

VerG

VerkOSG

VermG

VermV

VersE
Unabhängiger Finanzsenat

Universitätsgesetz 2002 BGBl I 2002/120

Unternehmensgesetzbuch BGBl I 2005/120

Urkundenhinterlegungsgesetz BGBl 1974/326

United Nations Commission on International Trade Law (Kommission der Vereinten Nationen für internationales Handelsrecht)

Übereinkommen der Vereinten Nationen über

Verträge über den Internationalen Warenkauf

BGBl 1988/96

unstreitig

unzutreffend

Unternehmensrechts-Änderungsgesetz 2008

BGBl I 2008/70

Urheberrechtsgesetz BGBl 1936/111

Urlaubsgesetz BGBl 1976/390

Unterhaltsschutzgesetz 1985 BGBl 452 (Wv)

Umsatzsteuer

Umsatzsteuergesetz 1972 BGBl 223

Haager Unterhaltsstatutsübereinkommen

BGBl 1961/293

und so weiter

unter Umständen

und viele(s) andere

Unterhaltsvorschußgesetz 1985 BGBl 451 (Wv)

Unabhängiger Verwaltungssenat

Bundesgesetz gegen den unlauteren Wettbewerb 1984 BGBl 448 (Wv)

Verordnung

vor allem

Versicherungsaufsichtsgesetz BGBl 1978/569

Vertragsbedienstetengesetz 1948 BGBl 86

Verbraucherbehörden-Kooperationsgesetz BGBl I 2006/148

Verbandsverantwortlichkeitsgesetz BGBl I 2005/151

Verwaltungsentlastungsgesetz BGBl 1925/277

Vereinsgesetz 2002 BGBl I 2002/66

Bundesgesetz über den erweiterten Schutz der Verkehrsopfer - Verkehrsopferschutzgesetz BGBl $1977 / 322$

Vermessungsgesetz BGBl 1968/306

Vermessungsverordnung 1994 BGBl 562

Versicherungsrechtliche Entscheidungssammlung 


\section{Abkürzungsverzeichnis}

\begin{tabular}{|c|c|}
\hline VersR & $\begin{array}{l}\text { (deutsches) Versicherungsrecht, Juristische Rund- } \\
\text { schau für die Individualversicherung }\end{array}$ \\
\hline verst & verstärkter \\
\hline VersVG & Versicherungsvertragsgesetz 1958 BGBl 1959/2 \\
\hline $\mathrm{VfGH}$ & Verfassungsgerichtshof \\
\hline VfSlg & $\begin{array}{l}\text { Sammlung der Erkenntnisse und wichtigsten Be- } \\
\text { schlüsse des Verfassungsgerichtshofes }\end{array}$ \\
\hline $\mathrm{vgl}$ & vergleiche \\
\hline $\mathrm{vH}$ & vom Hundert \\
\hline VIBOR & Vienna Interbank Offered Rate \\
\hline VJ & Verkehrsjurist des ARBÖ (Zeitschrift) \\
\hline VKG & Väter-Karenzgesetz BGBl 1989/651 \\
\hline VKI & Verein für Konsumenteninformation \\
\hline VKrG & Verbraucherkreditgesetz BGBl I 2010/28 \\
\hline & Vorarlberg, Vorarlberger \\
\hline VO & Verordnung(en) der EG \\
\hline VOEG & $\begin{array}{l}\text { Verkehrsopfer-Entschädigungsgesetz BGBl I } \\
2007 / 37\end{array}$ \\
\hline Vorbem & Vorbemerkungen \\
\hline & Die Versicherungsrundschau \\
\hline VRInfo & Information zum Verbraucherrecht \\
\hline & versus (gegen) \\
\hline VslgG & Versammlungsgesetz 1953 BGBl 98 \\
\hline VSPAG & $\begin{array}{l}\text { Vereinssachwalter- und Patientenanwaltsgesetz } \\
\text { BGBl 1990/156 }\end{array}$ \\
\hline VSPBG & $\begin{array}{l}\text { Vereinssachwalter-, Patientenanwaltschafts- und Be- } \\
\text { wohnervertretergesetz BGBl 1990/156 }\end{array}$ \\
\hline VStG & Verwaltungsstrafgesetz 1991 BGBl $52(\mathrm{Wv})$ \\
\hline VVDStRL & $\begin{array}{l}\text { Veröffentlichungen der Vereinigung der Deutschen } \\
\text { Staatsrechtslehrer }\end{array}$ \\
\hline VVG & Verwaltungsvollstreckungsgesetz 1991 \\
\hline $\mathrm{VwGH}_{w}$ & Verwaltungsgerichtshof \\
\hline VwSlg & $\begin{array}{l}\text { Erkenntnisse und Beschlüsse des Verwaltungsge- } \\
\text { richtshofes }\end{array}$ \\
\hline WaffG & Waffengesetz 1996 BGBl I 1997/12 \\
\hline & Wertpapieraufsichtsgesetz BGBl 1996/753 \\
\hline 3. WÄG & 3. Wohnrechtsänderungsgesetz BGBl 1993/800 \\
\hline $\begin{array}{l}\text { WBFG } \\
\text { wbl }\end{array}$ & $\begin{array}{l}\text { Wasserbautenförderungsgesetz } 1985 \text { BGBl } 148(\mathrm{Wv}) \\
\text { wirtschaftsrechtliche blätter, Zeitschrift für öster- } \\
\text { reichisches und europäisches Wirtschaftsrecht }\end{array}$ \\
\hline & Wohnungseigentumsgesetz 1975 BGBl 417 \\
\hline WEG 2002 & Wohnungseigentumsgesetz 2002 BGBl I 2002/70 \\
\hline WG & Wechselgesetz 1955 BGBl 49 \\
\hline
\end{tabular}




\section{Abkürzungsverzeichnis}

$\begin{array}{ll}\text { WGG } & \text { Wohnungsgemeinnützigkeitsgesetz BGBl 1979/139 } \\ \text { WGN 1997 } & \text { Erweiterte Wertgrenzen-Novelle 1997 BGBl I } \\ & \text { 1997/140 } \\ \text { WHG } & \text { Wachbediensteten-Hilfeleistungsgesetz BGB1 } \\ & \text { 1992/177 } \\ \text { WIPO } & \text { World Intellectual Property Organization } \\ \text { WKG } & \text { Wirtschaftskammergesetz 1998 BGBl I 1998/103 } \\ \text { WM } & \text { (deutsche) Zeitschrift für Wirtschafts- und Bank- } \\ & \text { recht, Wertpapier-Mitteilungen } \\ \text { wobl } & \text { Wohnrechtliche Blätter } \\ \text { Wr } & \text { Wiener } \\ \text { WrDiplKonv } & \text { Wiener Diplomatenkonvention BGBl 1966/66 } \\ \text { WRG } & \text { Wasserrechtsgesetz 1959 BGBl 215 idF WRG-No- } \\ & \text { velle 1990 BGBl 252 } \\ \text { WRInfo } & \text { WirtschaftsrechtsInfo } \\ \text { WRN 2006 } & \text { Wohnrechtsnovelle 2006 BGBl I 2006/124 } \\ \text { WRP } & \text { Wettbewerb in Recht und Praxis (deutsche Zeit- } \\ & \text { schift) } \\ \text { WTBG } & \text { Writtschaftstreuhandberufsgesetz BGBl I 1999/58 } \\ \text { WTBO } & \text { Wirtschaftstreuhänder-Berufsordnung BGBl } \\ & \text { 1955/125 } \\ \text { WuchG } & \text { Wuchergesetz 1949 BGBl 271 (Wv) } \\ \text { Wv } & \text { Wiederverlautbarung } \\ \text { WWSGG } & \text { Wald- und Weideservituten-Grundsatzgesetz BGBl } \\ & \text { 1951/103 } \\ \text { WWU } & \text { Wirtschafts- und Währungsunion } \\ \text { Z } & \text { Zahl, Ziffer } \\ \text { ZaDiG } & \text { Zahlungsdienstegesetz BGBl I 2009/66 } \\ \text { Zak } & \text { Zivilrecht aktuell } \\ \text { ZÄKG } & \text { Zahnärztekammergesetz BGBl I 2005/154 } \\ \text { ZAS } & \text { Zeitschrift für Arbeitsrecht und Sozialrecht } \\ \text { zB } & \text { zum Beispiel } \\ \text { ZBB } & \text { (deutsche) Zeitschrift für Bankrecht und Bankwirt- } \\ & \text { schaft } \\ \text { ZB1 } & \text { Zentralblatt für die juristische Praxis } \\ \text { ZER } & \text { Euro-Info, Blg zur Zeitschrift für Rechtsverglei- } \\ & \text { chung, Internationales Privatrecht und Europarecht } \\ \text { ZessRäG } & \text { Zessionsrechts-Änderungsgesetz BGBl I 2005/51 } \\ \text { ZEuP } & \text { Zeitschrift fur europäisches Privatrecht } \\ \text { ZfRV } & \text { Zeitschrift für Rechtrvergleichung, Internationales } \\ & \text { Privatrecht und Europarecht } \\ \text { ZfV } & \text { Zeitschrift für Verwaltung } \\ \text { ZfVB } & \text { Die administrativrechtlichen Entscheidungen des } \\ & \text { VwGH und die verwaltungsrechtlich relevanten } \\ & \end{array}$




\section{Abkürzungsverzeichnis}

Entscheidungen des VfGH in lückenloser Folge,

ZGB

ZGS

ZIK

ZinsRÄG

ZIP

zit

ZivMediatG

ZivRÄG

ZLB

ZNR

ZÖR

ZPMRK

$\mathrm{ZPO}$

ZRInfo

zT

ZTKG

zust

ZustG

zutr

ZVB

ZVglRWiss

ZVN 1983

ZVR
Beilage zur Zeitschrift für Verwaltung

(Schweizer) Zivilgesetzbuch

(deutsche) Zeitschrift für Vertragsgestaltung, Schuldund Haftungsrecht (bis 2008: Zeitschrift für das gesamte Schuldrecht)

Zeitschrift für Insolvenzrecht und Kreditschutz

Zinsenrechts-Änderungsgesetz BGBl I 2002/118

(deutsche) Zeitschrift für Wirtschaftsrecht und Insolvenzpraxis

zitiert

Zivilrechts-Mediations-Gesetz BGBl I 2003/29

Zivilrechts-Änderungsgesetz 2004 BGBl I 2004/91

Zeitschrift für Liegenschaftsbewertung

Zeitschrift für Neuere Rechtsgeschichte

Zeitschrift für öffentliches Recht

Zusatzprotokoll zur Konvention zum Schutze der

Menschenrechte und Grundfreiheiten

Zivilprozeßordnung RGBl 1895/113

ZivilrechtsInfo (ab 2006: Zak)

zum Teil

Ziviltechnikerkammergesetz 1993 BGBl 1994/157

zustimmend

Zustellgesetz BGBl 1982/200

zutreffend

Zeitschrift für Vergaberecht und Beschaffungspraxis (deutsche) Zeitschrift für vergleichende Rechtswissenschaft

Zivilverfahrens-Novelle 1983 BGBl 135

Zeitschrift für Verkehrsrecht 